\title{
Die großen wissenschaftlichen Leistungen von Stefan Vogel (1925-2015) Teil 4. Die Parfümblumen und ihre prächtigen Bestäuber
}

\author{
Anton Weber \& Günter Gerlach
}

\begin{abstract}
One of STEFAN VOGEL's ground-breaking contributions to the science of floral ecology was his discovery of perfume flowers (numerous orchids, as well as various genera and species within Araceae, Gesneriaceae, Euphorbiaceae, and Solanaceae) and their pollination by highly specialized male euglossine bees (also known as orchid bees, Apidae-Euglossini). Only pollen and nectar had been known as floral rewards for more than 250 years until Vogel eventually discovered a unique new type of floral reward particularly sought after by male bees, turning out as one of the most fascinating interactions between plants and animals. Apart from botanical features, VOGEL also thoroughly investigated the morphology, anatomy, and behavior of the involved euglossine bees and presented a sound explanation for the peculiar structures on the fore, mid, and hind legs as adaptations for scent-collecting of the males.
\end{abstract}

\section{Zusammenfassung}

Mit der Entdeckung der „Parfümblumen“ (zahlreiche Orchideen sowie vereinzelt Gattungen und Arten der Araceae, Gesneriaceae, Euphorbiaceae und Solanaceae) und ihre Bestäubung durch hochspezialisierte, männliche Prachtbienen (Apidae-Euglossini) hat Stefan Vogel einen bahnbrechenden Beitrag zur Blütenökologie geleistet. Nachdem mehr als 250 Jahre lang nur Pollen und Nektar als florale Beköstigungsmittel bekannt waren, hat er ein völlig neuartiges florales Sammelgut exklusiv für männliche Prachtbienen entdeckt und eine der faszinierendsten Tier-Pflanzen-Interaktionen aufgeklärt. Neben den botanischen Aspekten hat Vogel auch wesentlich zur Kenntnis und zum Verständnis der Morphologie, Anatomie und Verhaltensbiologie der Prachtbienen beigetragen und die speziellen Strukturen an den Vorder-, Mittel- und Hinterbeinen als Anpassungen an das Duftsammeln der männlichen Tiere deuten können.

\section{Die verschlungenen Pfade der Entdeckung} Im Zusammenhang mit seinen Studien über Blütendüfte und ihren Bildungsstätten, den Osmophoren, stellte Vogel fest, dass die bisher als Futtergewebe interpretierten Blütenstrukturen mancher neotropischer Orchideen (Catasetum, Stanhopea u. a.) Osmophoren darstellen (vgl.

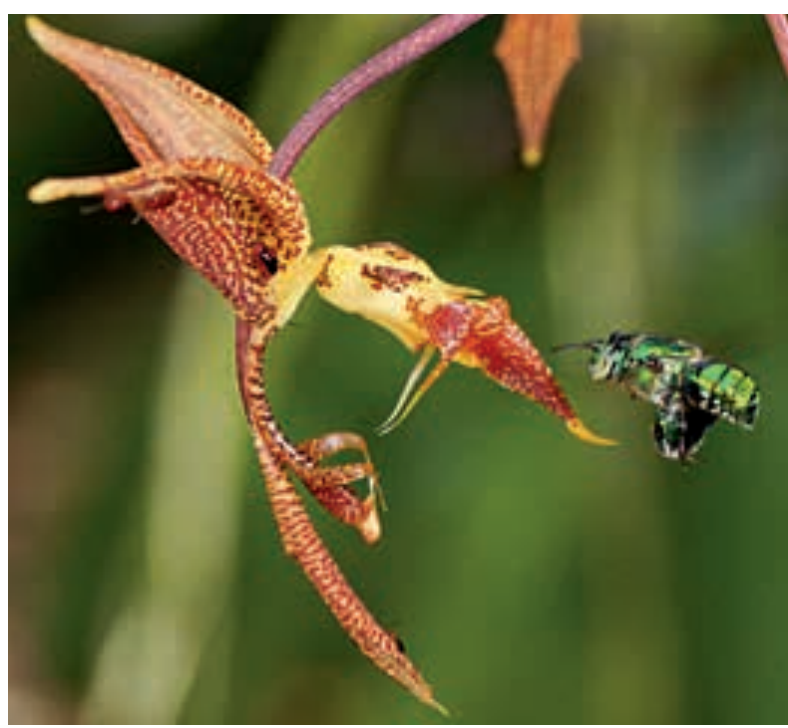

Abb. 1. Ein Männchen von Euglossa tridentata im Anflug an eine Blüte von Gongora sp. (Orchidaceae) (Costa Rica).
Weber \& Steinecke 2017). Er erwähnte dies bereits in seiner klassischen Studie „Düftdrüsen im Dienste der Bestäubung“ (Vogel 1963a) und widmete diesem Faktum eine weitere, detaillierte Studie mit dem Titel „Das sexuelle Anlockungsprinzip der Catasetineen- und Stanhopeen-Blüten und die wahre Funktion ihres sogenannten Futtergewebes"

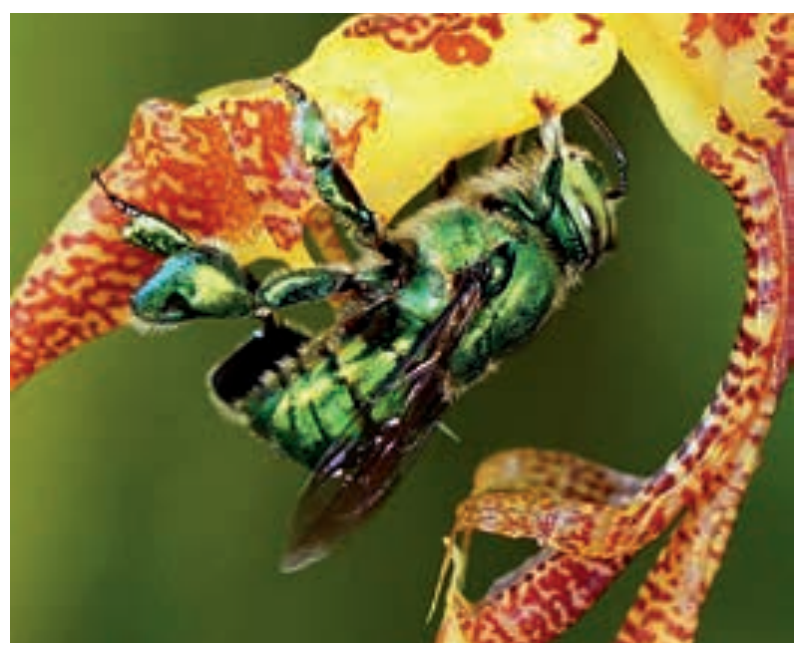

Abb. 2. Nach der Landung wischt das Bienenmännchen mit den Tarsalbürsten der Vorderbeine den Duftstoff von der (topographischen) Unterseite des Labellums ab. Am Hinterbein ist die helmförmig aufgeblähte Tibia gut zu erkennen. 
(Vogel 1963b). Diese Arbeit behandelte auch in ausführlicher Form die zoologischen Aspekte, d. h. die Morphologie und das Verhalten der Bienen (Vogel 1963b). Anlässlich seiner Kolumbienreise $1955 / 56$ hatte er bereits beobachtet, dass die Blüten von Catasetum-Arten von Prachtbienen besucht werden und zwar ausschließlich von Männchen (Vogel in Troll 1960: 91): „... werden Bestäuber allein durch den Duft der Blüte angelockt, welcher, da sich nur Euglossa-Männchen einfinden, vermutlich den weiblichen Sexualgeruch imitiert. Es besteht darin eine bemerkenswerte Analogie zu dem Verhalten der Ophrys-Arten." Ähnliche Beobachtungen hatten später und unabhängig amerikanische Forscher im Jahre 1959 gemacht und 1961 publiziert (Dodson \& Frymire 1961). Sie beschrieben, dass die männlichen Prachtbienen beim Besuch der Blüten von Catasetum macroglossum in einen rauschartigen Zustand verfallen: „... the bees [Eulaema cingulata] become intoxicated and fight among themselves for the right to enter a given flower, ....the bees were not visiting the flowers for nectar or to gnaw the fleshy material inside but come as if drawn to the musky scent of the flowers to be drugged...", p. 134). Trotz ähnlicher Beobachtungen bei anderen Orchideen konnten sich Dodson \& Frymire (1961) darauf keinen Reim machen. Sätze wie „This peck at the picture left us with far more questions than answers“, oder „A great many observations need to be accumulated before the rest of the picture will begin to become clear" in den Schlussfolgerungen bezeugen ihre Ratlosigkeit.

Es war Stefan Vogel vorbehalten, den Sachverhalt aufzuklären. Allerdings gelang es auch ihm nicht, dies mit einem Schlag zu tun. Nachdem ihm klar geworden war, dass die Blüten Osmophoren (und kein Futtergewebe) besitzen, nahm er zunächst an, dass es sich bei diesen Orchideen-Blüten um Sexualtäuschblumen nach dem Muster von Ophrys handeln müsse (Vogel 1963b). Die Wende brachte eine weitere Reise nach Südamerika (1964). Hier trug er weiteres Material zusammen und machte ausgedehnte Beobachtungen. Auch fand er heraus, dass die Bestäubung durch männliche Prachtbienen nicht auf die Orchideen beschränkt ist, sondern auch bei dikotylen Pflan- zen (Gloxinia perennis, Gesneriaceae) vorkommt. In der klassischen Studie „Parfümsammelnde Bienen als Bestäuber von Orchideen und Gloxinia“ (Vogel 1966) publizierte er seine Ergebnisse und legte eine Hypothese vor, die bis heute Bestand hat und durch viele neue Beobachtungen und das Bekanntwerden neuer Fälle erhärtet worden ist.

Die Erkenntnisse, die in den letzten Jahren und Jahrzehnten gewonnen wurden, sind $\mathrm{u}$. a. bei Vogel (1999), Gerlach (1999, 2015), Singer \& Gerlach (2002) und (mit Schwerpunkt auf den zoologischen Aspekten) ELTz $(2003,2010)$ in allgemeinverständlicher Form zusammengefasst. Die letztzitierte Arbeit enthält auch lesenswerte Überlegungen zur phylogenetischen Entstehung der Parfümblumen und Prachtbienenbestäubung, die Vogel (1999) als noch im Dunkeln liegend bezeichnet hatte.

\section{Vogels Erkenntnisse und Thesen}

Sie lassen sich in aller Kürze folgendermaßen zusammenfassen:

(1) Die Parfümblumen bieten weder Pollen noch Nektar, sondern Duftstoffe als florales Lock- und Belohnungsmittel an. Doch ist zu betonen, dass Parfümblumen nicht einfach mit duftenden Blüten gleichgesetzt werden dürfen. Normaler Blütenduft dient nur der (Fern-)Anlockung von Bestäubern, der Duft der Parfümblumen fungiert hingegen zusätzlich und gleichberechtigt als Sammelgut. Der Duftstoff (komplexe Mischung verschiedener, chemischer Verbindungen in feinen Tröpfchen sezerniert und flüchtig) haben somit eine Doppelfunktion: olfaktorische Anlockung plus Sammelgut.

(2) Die Duftstoffe locken ausschließlich männliche Prachtbienen (Euglossini) an und werden von diesen aktiv gesammelt, in speziellen Organen gespeichert und später aktiv mit Hilfe spezieller Strukturen an der Flügelbasis und durch Ventilieren der Flügel versprüht. Da die Prachtbienen eine rein neotropische Bienengruppe darstellen, sind Parfümblumen auf die Neotropen beschränkt.

(3) Das Sammeln wird durch spezialisierte Organe der Vorderbeine ermöglicht: die Tarsalglieder 
(Endglieder der Beine) tragen quastenförmige Anhänge, mit denen die tröpfchenförmigen Duftstoffe in charakteristischen Wischbewegungen aufgenommen werden.

(4) Beim kurzen Abheben vom Sammelort wird der Duftstoff über die Mittelbeine zu den Hinterbeinen transferiert („Rückladen“) und dort in den sog. Tibialorganen (aufgeblähte, von feinen Trabekeln, Drüsen und Muskeln durchzogenen Hinterschienen) gespeichert, sodass sich ein artspezifischer „männlicher Körpergeruch“ ergibt.

(5) Die Bienenmännchen setzen sich nach Beendigung des Sammelaktes auf erhöhte Stellen („Ansitz“) und schlagen im Sitzen mit gestreckten Beinen mit den Flügeln. Dadurch wird der aus verschiedenen Blüten zusammengetragene Duft aus den Tibialorganen nach außen transferiert und versprüht.

(6) Das Versprühen des Duftstoffes dient allem Anschein nach der Markierung des Territoriums bzw. der Schwarmbahn der Bienenmännchen.

\section{Vorkommen der Parfümblumen}

Vogels frühe Beobachtungen (Vogel 1966a) bezogen sich in erster Linie auf die Orchideen, wobei er feststellen konnte, dass die Subtriben Catasetinae und Stanhopeinae (Gongorinae) zur Gänze und die Subtriben Lycastinae, Zygopetalinae teilweise durch den Besitz von Parfümblumen charakterisiert sind. Aus den Araceen konnte er für Spathiphyllum (namentlich S. cannifolium) Parfümproduktion nachweisen. Aus der großen Gruppe der Dikotyledonen konnte er ebenfalls nur eine einzige Gattung/Art nennen und genauer beschreiben: Gloxinia perennis aus der Familie der Gesneriaceen.

Heute wissen wir über die Verbreitung etwas mehr: bei den Orchideen sind weitere Gruppen dazugekommen, und man schätzt, dass bis zu 6 Prozent bzw. rund 750 Arten der neotropischen Orchideen Parfümblumen besitzen. Als die wichtigsten Gattungen wären zu nennen: Anguloa, Catasetum, Coryanthes, Dichaea, Gongora, Notylia und Stanhopea. Bei den Araceen ist vor allem die

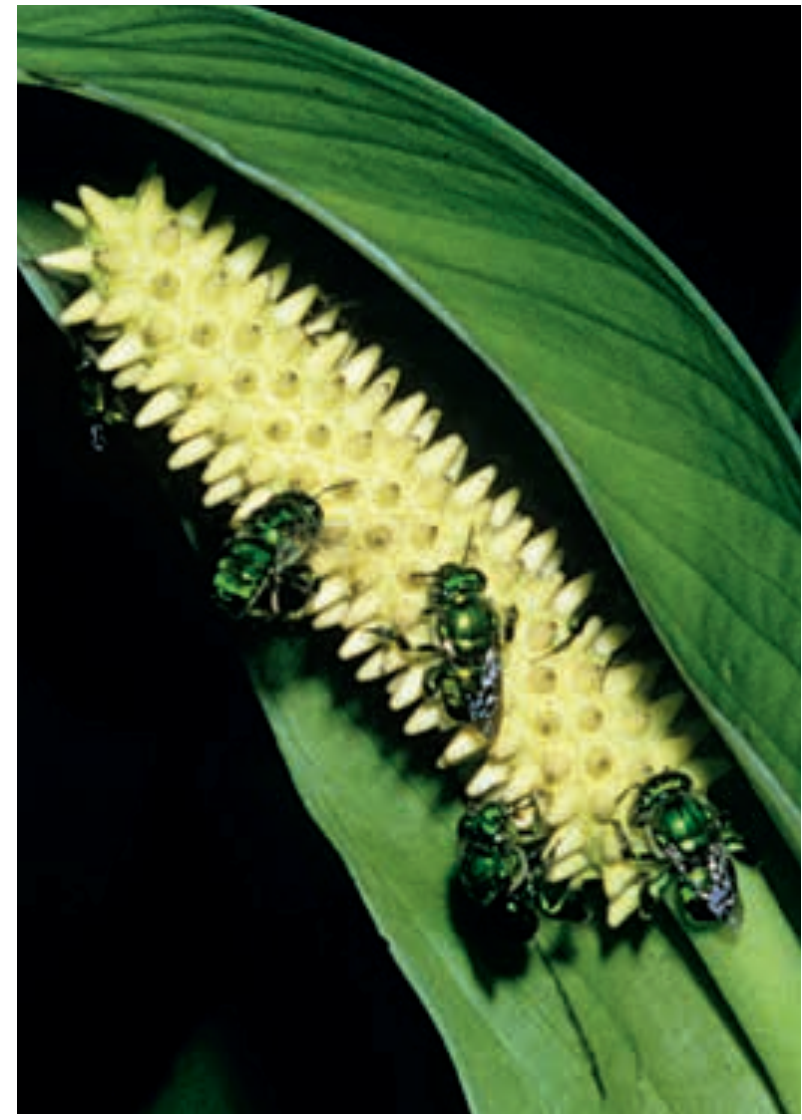

Abb. 3. Blütenstand von Spathiphyllum lancifolium (Araceae) mit mehreren Prachtbienen-Männchen (Euglossa chlorina und E. variabilis) (Venezuela).

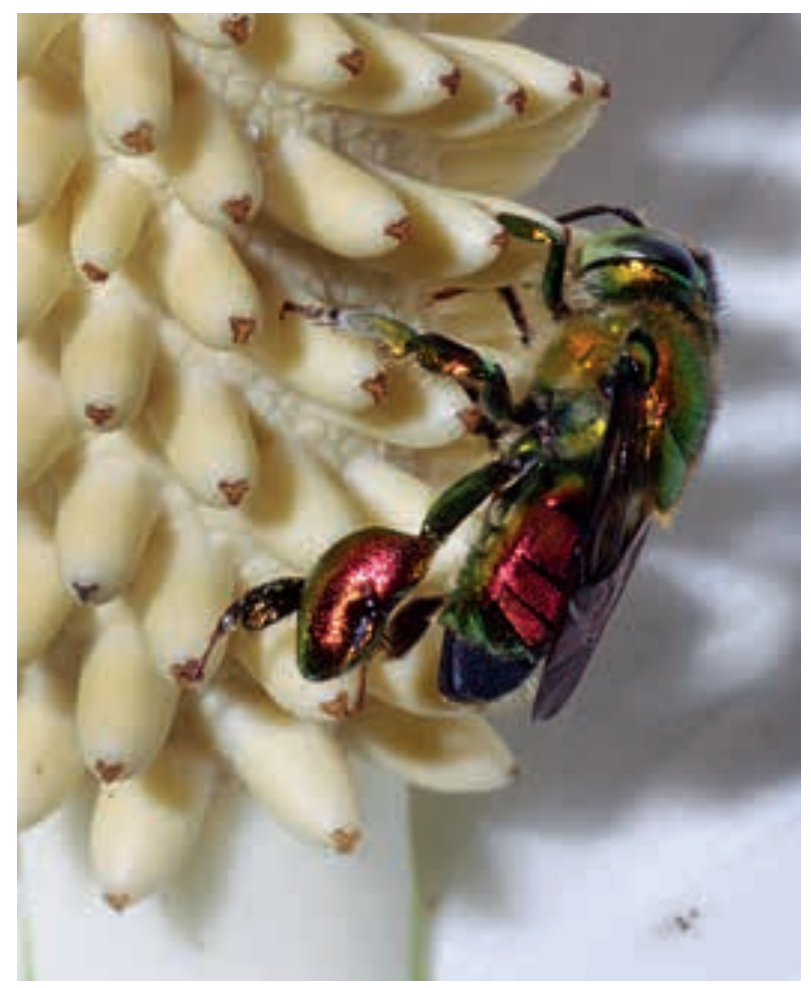

Abb. 4. Ein Prachtbienen-Männchen (Euglossa alleni) an einem Blütenstand von Spathiphyllum friedrichsthalii (Araceae) (Costa Rica). 
große Gattung Anthurium (> 1000 Arten) hinzugekommen, in der ein beträchtlicher Anteil der Arten Parfümblumen bildet (Dressler 1968; Schwerdtfeger et al. 2002). Daneben spielt auch die Gattung Spathiphyllum eine wichtige Rolle. Wie sich die Duftbouquets verschiedener Anthurium- und Spathiphyllum-Arten unterscheiden und als Mittel der reproduktiven Isolation eingesetzt werden, haben Hentrich et al. (2010) untersucht.

Bei den Gesneriaceen scheinen neben Gloxinia perennis einige weitere Vertreter der Familie Parfümblumen zu besitzen. Neben den beiden anderen Arten der neu- und engdefinierten Gattung Gloxinia (RoAlson et al. 2005a, b, BogGAN 2006) steht vor allem die Gattung Monopyle im Verdacht, Parfümblumen zu besitzen (Vogel et al. 2002). Näheres ist aber nicht bekannt. Bei den Dikotyledonen sind in der Zwischenzeit noch zwei weitere Familien mit Parfümblumen bekannt geworden: die Euphorbiaceen und die Solanaceen. In beiden Fällen ist es jeweils nur eine einzige Gattung, und auch hier nur ein kleiner Teil der Arten, welche Parfümblumen besitzen. Bei den Euphorbiaceen ist es die Gattung Dalechampia, in der etwa ein Dutzend der über 100 Arten Parfümblumen aufweist. Ihre Entdeckung und Erforschung erfolgte durch Armbruster \& Webster (1979; Details zur Erforschungsgeschichte siehe WeBsteR \& Armbruster 1991). Die Arten mit Parfümblumen verteilen sich innerhalb der Gattung auf drei Sektionen: sect. Cremophyllum (mit der bekanntesten Art D. spathulata, die häufig in den Glashäusern botanischer Gärten kultiviert wird), sect. Rhopalostylis, und sect. Dalechampia, woraus zu schließen ist, dass sich Parfümblumen innerhalb der Gattung dreimal unabhängig voneinander entwickelt haben.

Bei den Nachtschattengewächsen sind es die Arten von Solanum sect. Pachyphylla (früher Gattung Cyphomandra) mit einigen wenigen Parfümblumen-Arten (die bekannte Baumtomate, Solanum betacea, gehört allerdings nicht dazu; obwohl sie sehr intensiv und angenehm duftet, besitzt sie Pollenblumen, die äußerlich und funktionell jenen der Kartoffelpflanze ähnlich sind). An ihrer
Entdeckung bzw. Aufklärung des Bestäubungsmechanismus trägt wiederum STEFAN Vogel maßgeblichen Anteil (Sazima, Vogel et al. 1999). Die Blüten der betreffenden Solanum-Arten sind dadurch besonders bemerkenswert, als hier die Staubblätter, welche die Osmophoren tragen, einen Blasebalg-Mechanismus (im Gegensatz zum häufigen und einfachen Streukegel, aus denen die Bestäuber den Pollen herausvibrieren) aufweisen: Wischt eine Prachtbiene das Parfüm von der aufgeblasenen Anthere ab, übt sie einen Druck auf diese aus. Dadurch stößt die Anthere an ihrer Spitze eine kleine Pollenwolke aus und stäubt die Biene ein. Erst vor wenigen Jahren wurde bekannt, dass Parfümblumen bzw. Bestäubung durch männliche Prachtbienen auch bei einem Vertreter der basalen Angiospermen (Annonaceae: Unonopsis stipitata) vorkommt (TEICHERT et al. 2009).

Zusammenfassend lässt sich sagen, dass die Orchideen bei weitem die Hauptmasse der Gattungen/Arten mit Parfümblumen stellen. Die zweitgrößte Gruppe sind die Araceen, in der viele Arten der beiden Gattungen Anthurium und Spathiphyllum Parfümblumen besitzen. Bei den Dikotyledonen sind es nur sehr wenige Familien und auch hier nur eine oder ganz wenige Gattungen und Arten, deren Blüten Parfüm darbieten und von Prachtbienen bestäubt werden.

\section{Die Prachtbienen und \\ ihre Sammeltätigkeit}

Die Prachtbienen stellen eine Untergruppe der Echten Bienen (Apidae) dar. Ihr Name, Euglossini, nimmt Bezug auf den langen Rüssel ( glossa $=$ Zunge), der länger als der Körper sein kann und beim Fliegen zurückgeklappt und so zwischen den Beinen zu liegen kommt. Zahlreiche Blütenpflanzen der Neotropis haben sich an die oft extrem langen Zungen der Euglossini beiderlei Geschlechts angepasst, sie verbergen z. B. ihren Nektar in tiefen Spornen. Wie schon erwähnt, kommen sie ausschließlich in den Neotropen (Mittelamerika und tropisches Südamerika) vor. Die nahezu-250 Arten werden 5 Gattungen zugeordnet: Euglossa, Eulaema, Eufriesea, Exaerete und Aglae. Außer Eulaema zeichnen sich alle durch eine metallische Färbung in Grün-, Blau-, Rot- und Goldtönen aus. Die 


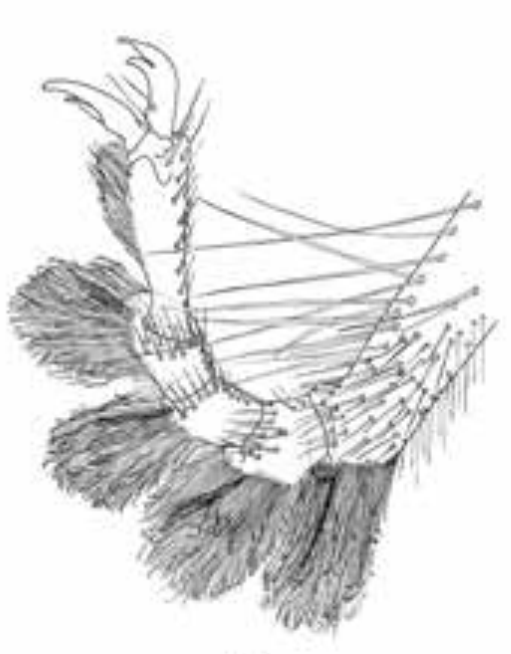

a

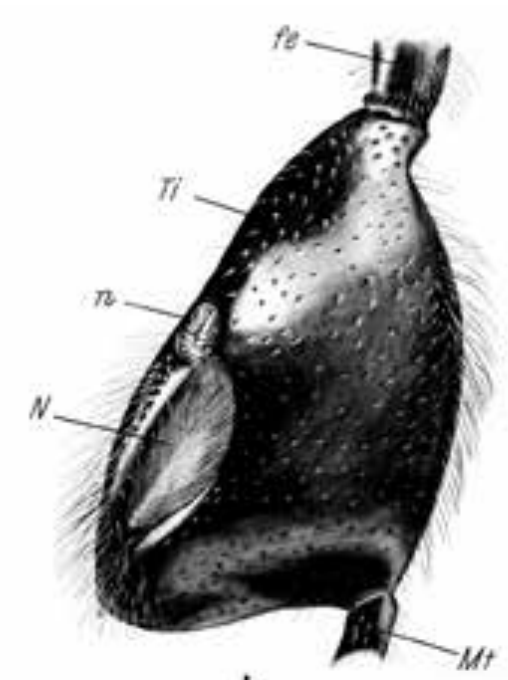

b

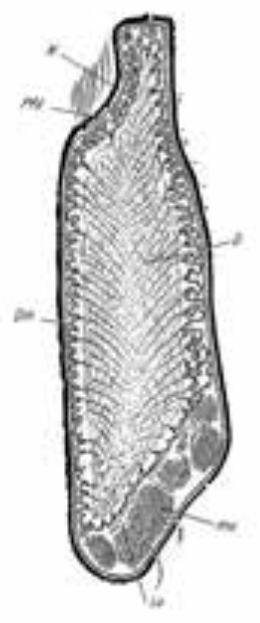

C
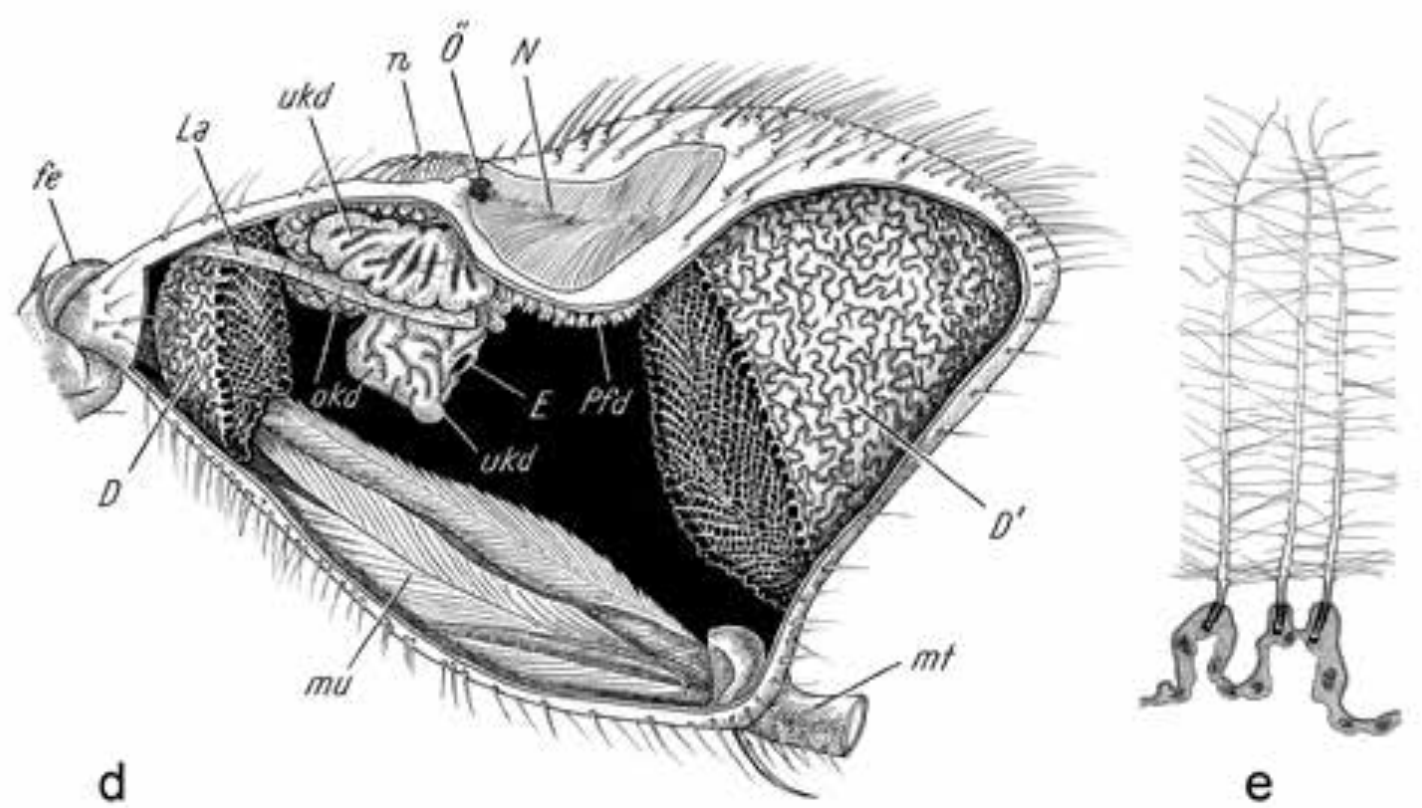

Abb. 5. a Tarsalglieder mit Tarsalquasten („Tarsalbürste“) von Eulaema sp. (als E. longipennis); b Tibialorgan von Euglossa analis (als E.bicolor); c, d Schematischer Quer- und Längsschnitt durch das Tibialorgan einer Prachtbiene; e Isolierte Trabekel aus dem Inneren des Tibialorgans. Ti Tibialorgan, N große Pfanne (Napf), n kleine Pfanne, D, D‘ innerer, von Trabekeln ausgefüllter Hohlraum des Tibialorgans, mu Muskeln, okd, ukd oberer bzw. unterer Drüsenkomplex (aus Vogel 1963b, Abb. 9/V [a], 9/III [b] und Vogel 1966, Abb. 9/I [c]), 8 [d] und 9/II [e]).

Eulaema-Arten sehen hingegen großen Hummeln recht ähnlich. Sie besitzen einen pelzigen Körper, einen schwarz-orange geringelten Hinterleib, und sind größer als die übrigen Prachtbienen. Vorweg ist festzuhalten, dass die Prachtbienen sich so verhalten, wie andere Bienen auch: die Männchen und Weibchen saugen zum Zwecke der Energieaufnahme Nektar an diversen Blüten und die Weibchen sammeln Pollen für die Brut. Dabei kommt es auch zur Pollenübertragung und Bestäubung. Das Besondere ist, dass die Männchen aber noch eine zusätzliche Strategie entwickelt und dafür besondere Anpassungen im Körperbau, nämlich Sonderbildungen an allen drei Beinpaaren, entwickelt haben: das Duftsammeln. Die Duftquellen sind verschiedener Natur und betreffen nicht nur Blüten. Letztere, die Parfümblumen, sind aber hier von besonderem Interesse.

Die Duftsubstanzen werden von speziellen Gewebebezirken, den Osmophoren, sezerniert und in Form feiner Tröpfchen an der Oberfläche 
abgelagert. Dort werden sie von den männlichen Bienen in charakteristischen Bewegungen aufgewischt. Dafür besitzen die Bienenmännchen besondere Strukturen an den Vorderbeinen: quasten- oder pinselförmige Anhänge an den fünf Tarsalgliedern, die zusammen die Tarsalbürste bilden. Die Quasten saugen die Duftstoffe auf und halten sie kapillar fest. Sind die Quasten vollgesaugt, fliegt die Biene kurz ein paar Zentimeter auf und streift die flüssigen Duftstoffe mit einer speziellen Vorrichtung an den Mittelbeinen ab: die basale Hinterkante des Metatarsus (das auf die Tarsen proximal folgenden Beinglied) weist eine mehr oder weniger tiefe Kerbe auf, in deren Bereich zwei Arten von Beinhaaren vorhanden sind: normale, dünne Borsten und einige stark verdickte, wellenförmig verbogene Dornen, die eine Art Rechen bilden. Zieht die Biene die vollgesogenen Tarsalquasten durch den Rechen, hält dieser die Duftstoffe zurück.

Auf diesen Vorgang folgt unmittelbar, also noch während des Fluges über der Duftquelle, ein zweiter: die Hinterbeine werden nach vorne gezogen, sodass sich der Rechen und die Oberseite des sog. Tibialorgans berühren. Um zu verstehen, was im Folgenden passiert, müssen wir den Bau des Tibialorgans etwas näher kennenlernen. Es ist ein stark aufgeblähtes Beinglied, nämlich jenes Gliedes, das körperwärts auf den Metatarsus folgt. Es ist im Prinzip ein Hohlorgan, dessen Inneres fast vollständig von feinen, fiederförmig verästelten Chitinhaaren („Trabekeln“) ausgefüllt ist. Der freibleibende Raum wird von den Beinmuskeln und von zwei Drüsenaggregaten eingenommen. Nach außen hin gibt es an der Oberseite zwei Öffnungen: eine große, etwas eingetiefte Öffnung, die von ringförmig angeordneten feinen Haaren verschlossen ist (die Große Pfanne) und eine kleine (die Kleine Pfanne), die durch Schuppen verschlossen ist. Berühren sich der Rechen und das Tibialorgan, werden die Duftstoffe vom Rechen über die große Pfanne kapillar in das Innere des Tibialorgans gesaugt und dort gespeichert. Die Drüsen bzw. ihre Sekrete dienen vermutlich dazu, dem Duftstoffgemisch fetthaltige Substanzen zuzusetzen, sodass es stabilisiert bzw. seine Flüchtigkeit herabgesetzt wird. Mit anderen Worten, es wird ein art-spezifisches Parfüm gebildet.

Ist der Prozess der Rückladung des Blütenduftes abgeschlossen, landet das Bienenmännchen wieder auf der Blüte und wischt erneut das duftproduzierende Blütenareal ab. Der Prozess des Abwischens und des Rückladens wird so lange fortgesetzt, bis das Tier erkennt, dass es genug von der Duftquelle gesammelt hat. Dann fliegt die Biene weg. Damit stellt sich die Frage, wozu das Ganze? Auch dafür hat Stefan Vogel eine bis heute unwidersprochene Antwort geliefert. Nach dem Verlassen der Blüte setzen sich die Bienenmännchen bevorzugt auf erhöhte Stellen (Ansitz) und beginnen mit den Flügeln zu ventilieren. Dabei werden offensichtlich die gespeicherten Duftstoffe, vermutlich über die kleine Pfanne, aus dem Tibialorgan herausgesaugt und versprüht. Die Männchen markieren so olfaktorisch das Territorium ihrer Balzflüge, in das dann die Weibchen einfliegen bzw. aus dem männliche Konkurrenten vertrieben werden. Das Weibchen erkennt aus dem arttypischen versprühten Parfüm das fitteste der Männchen und lässt es zur Kopulation zu. Tiere mit wenig Erfahrung und somit unvollständigem Duftstoffgemisch werden dagegen verschmäht, es besteht Damenwahl (female choice). Damit spielen die Parfümblumen eine essentielle Rolle im Fortpflanzungszyklus ihrer Bestäuber.

Abschließend muss man bewundernd feststellen, dass der Botaniker Stefan Vogel nicht nur ein neues florales Attraktionsmittel bei Blüten in zunächst drei Verwandtschaftskreisen entdeckt hat, sondern bewundernswerte Beiträge zur Zoologie geleistet hat. Er hat nicht nur den Vorgang des Duftsammelns, des Rückladens und des Speicherns in allen Details aufgeklärt und dokumentiert, sondern auch eine musterhafte Beschreibung und funktionelle Erklärung der mannigfaltigen morphologischen Besonderheiten der männlichen Prachtbienen geliefert. Die diesbezüglichen Zeichnungen gehören zu den besten und instruktivsten, die je über die Morphologie und Anatomie von Bienen publiziert worden sind. Ohne die $\mathrm{Zu}$ sammenschau der botanischen und zoologischen 
Aspekte wäre wohl die Deutung der ökologischen Zusammenhänge des Parfümblumensyndroms nicht möglich gewesen.

\section{Zusammensetzung der Duftstoffe}

Stefan Vogel hat nicht nur bahnbrechende Beobachtungen in der freien Natur gemacht, sondern betätigte sich auch im Labor als Naturstoffchemiker. Zu seiner Zeit waren die analytischen Methoden noch recht bescheiden und beschränkten sich im Wesentlichen auf die Dünnschichtchromatografie. Heute mutet dieses Verfahren recht antiquiert an und wird durch leistungsfähigere und präzisere Analyseverfahren wie die GC/ MS (gekoppeltes System aus Gaschromatograf und Massenspektrometer) ersetzt. Schon damals stellte sich heraus, dass die Duftkomposition bei Parfümblumen oft sehr einfach war und zuweilen nur aus einem einzigen Duftstoff bestand. Im Gegensatz dazu wären die Düfte der Rosen zu nennen, die manchmal aus mehr als 100 Komponenten zusammengesetzt sind. Eine wichtige Rolle bei den Parfümblumen spielen die Monoterpene, sie finden sich in fast allen Parfümblumen. Hier sind vor allem $\alpha$-Pinen, $\beta$-Pinen, Limonen und Eucalyptol (1,8-Cineol) anzutreffen. Konzentrationen von mehr als 80\% Eucalyptol sind keine Seltenheit. Verschiedene Ester, die sich oft durch einen sehr fruchtigen Duft charakterisieren lassen, sind ebenfalls vertreten. Darunter befinden sich Benzylacetat (Himbeerbonbons), Methylsalicylat (Wintergrünöl, erinnert an manche Kaugummis), Methylcinnamat (Zimtduft), Benzylbenzoat (fast geruchlos), Phenylethylacetat (schwerer intensiver Duft), sowie die dazugehörigen Alkohole und Aldehyde wie Benzylalkohol oder Benzaldehyd (Bittermandel). Häufig sind verschiedene Sesquiterpene zu finden, die sich nur mit großer Mühe identifizieren lassen, weil ihre Massenspektren sich sehr ähneln. Phenylpropanderivate wie Vanillin, Zimtaldehyd und vor allem Eugenol (Gewürznelke) gehören ebenfalls zu den häufiger anzutreffenden Duftstoffen. An stickstoffhaltigen organischen Verbindungen wurde vor allem das heterocyclische Indol gefunden, welches einen unangenehmen Duft verströmt. Skatol (3-Methylindol), verantwortlich für den typischen Gestank von Fäkalien, konnte
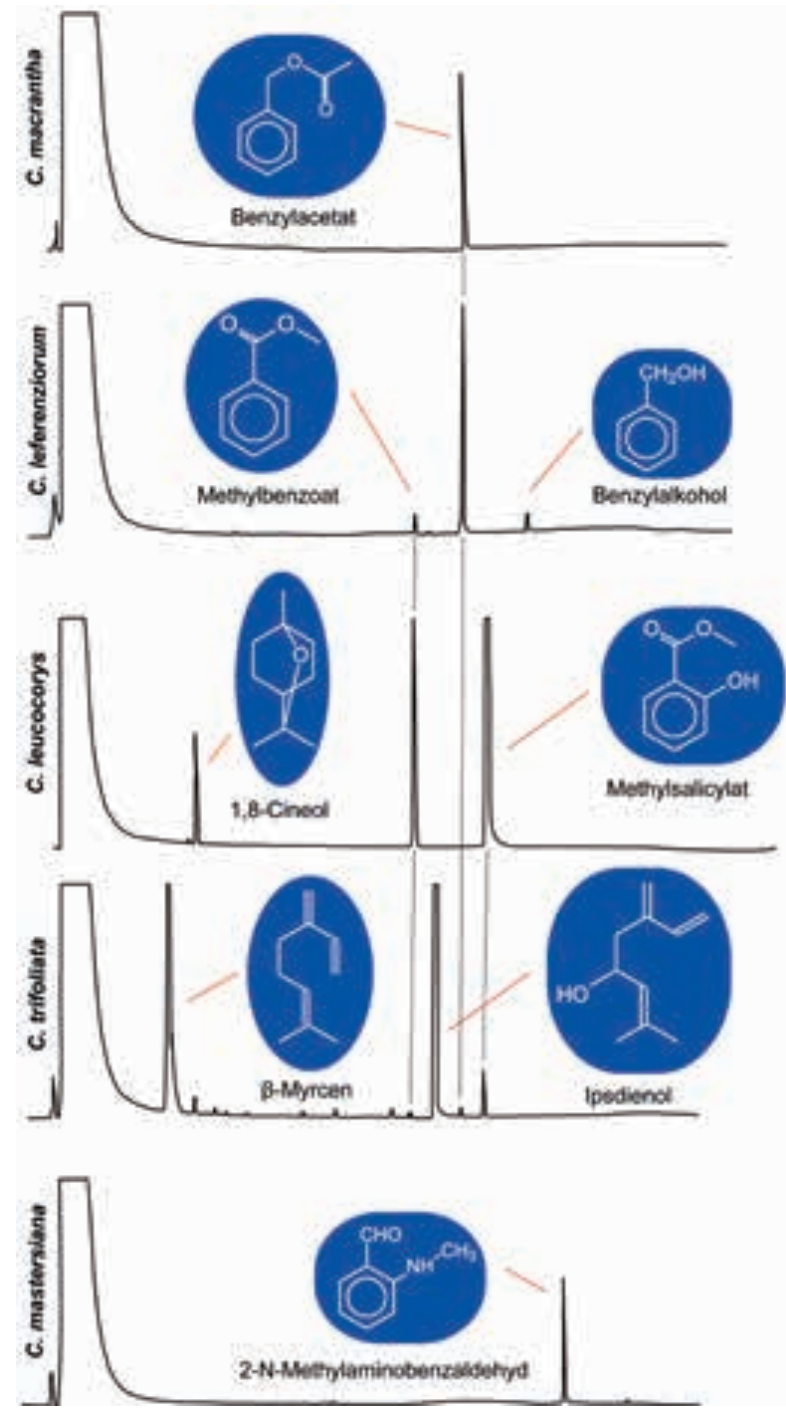

Abb. 6. Zusammensetzung der Blütendüfte verschiedener Coryanthes-Arten (Orchidaceae). Haupt- oder alleinige Komponenten mit Strukturformel (aus Gerlach 2015, Abb. S. 45).

bei ein paar wenigen Parfümblumen nachgewiesen werden, so vermag ein einziges blühendes Solanum melissarum die Luft eines ganzen Gewächshauses zu verpesten.

Da die von den Parfümblumen abgegebenen Duftstoffe nicht nur der Anlockung, sondern auch der Belohnung dienen, kamen schon kurz nach der Entdeckung des Syndroms Wissenschaftler auf die Idee, diese Stoffe als Köder zu benutzen. Dabei stellte sich heraus, dass nicht alle der in einem Blütenduft gefundenen Substanzen attraktiv wirken. Mit Stoffen wie Eucalyptol, Methylsalicylat, Eugenol u. a. m. lassen sich viele verschiedene Prachtbienenarten anlocken, andere 
wie $\beta$-Pinen wirken hingegen nicht. Mischungen von verschiedenen Komponenten schränken das angelockte Spektrum der Prachtbienenarten ein. Durch geschickte Kombination einer attraktiven Substanz „A“ mit verschiedenen Substanzen „B“,"CC“ etc., die selbst nicht attraktiv sein müssen, kann man erreichen, dass von den ursprünglich von Substanz „A“ köderbaren Prachtbienenarten nur noch wenige, wenn nicht gar nur eine Art, auf das Gemisch positiv reagieren. Orchideen weisen geringe Inkompatibilität gegenüber fremdem Pollen auf (deshalb die vielen Orchideenhybriden im Blumenhandel). Für die Artintegrität sind vielmehr externe Faktoren verantwortlich. Zwischen den Gattungen besteht in der Natur eine mechanische Barriere: die verschiedenen Gattungen platzieren ihre Pollinarien an verschiedenen Teilen des Bienenkörpers (vgl. Abb. 17 und 24). Verschiedene, im gleichen Areal vorkommende Arten einer Gattung unterscheiden sich aber in der Duftkomposition (Abb. 6). Sie locken verschiedene Prachbienenarten an und verhindern somit ihre Hybridisierung. Kurz: verschiedene Gattungen sind mechanisch getrennt, während verschiedene Arten einer Gattung sich durch ihren Blütenduft unterscheiden.

\section{Die Parfümblumen von Gloxinia perennis und ihre Bestäuber}

Gloxinia perennis war die erste (von Vogel 1966 entdeckte) und lange Zeit einzige Art der Dikotyledonen, deren Blüten als Parfümblumen einzustu-

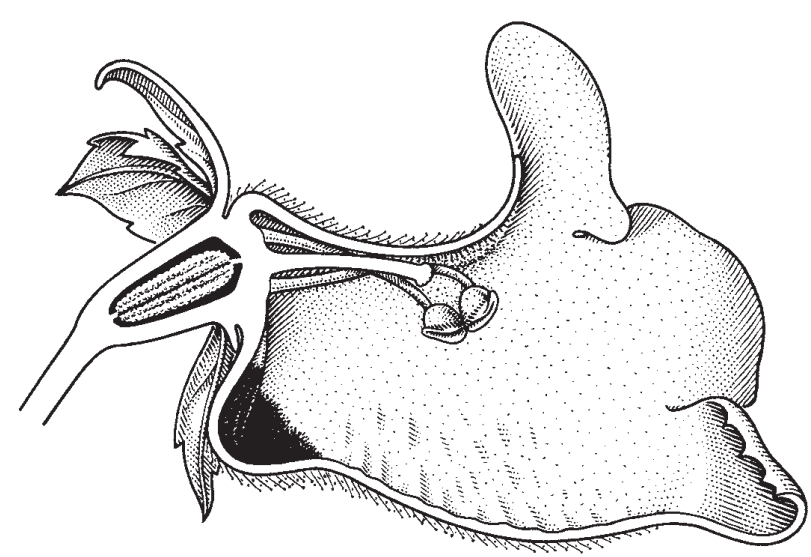

Abb. 7. Gloxinia perennis (Gesneriaceae). Blüte im Längsschnitt. Osmophor an der Blütenbasis schwarz (aus Vogel 1966, Abb. 12a).

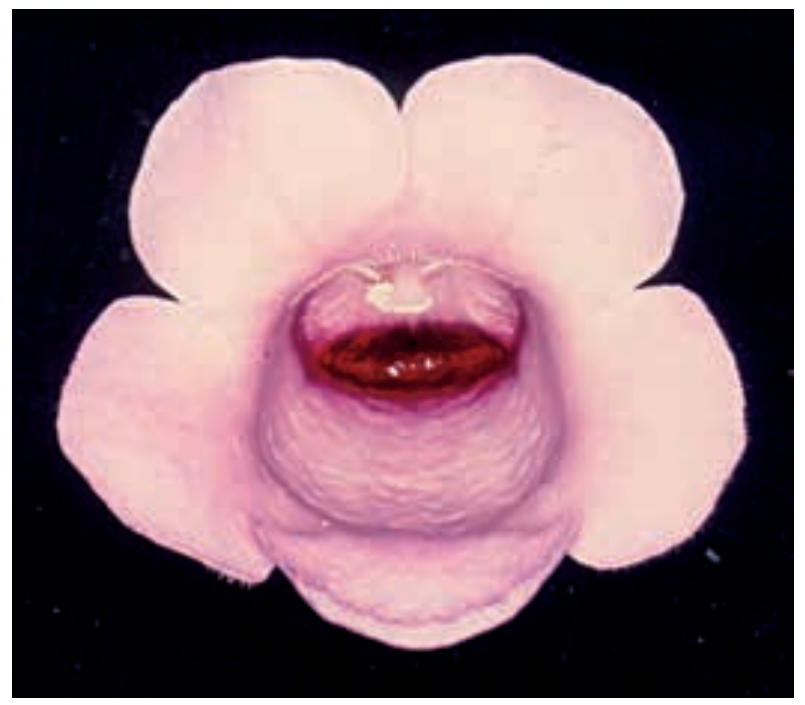

Abb. 8. Gloxinia perennis. Blüte in Vorderansicht, Osmophor dunkelviolett.

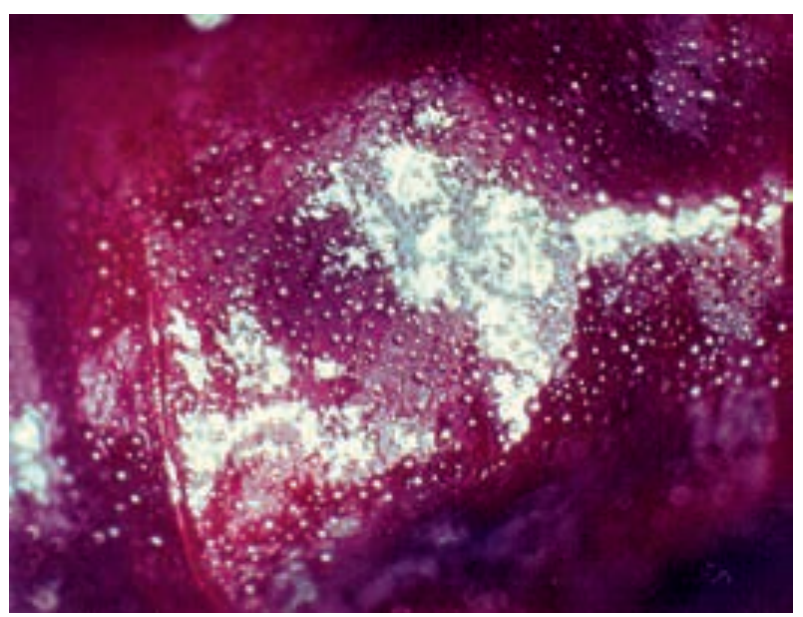

Abb. 9. Gloxinia perennis. Oberfläche des Osmophors mit Tröpfchen der Duftstoffe.

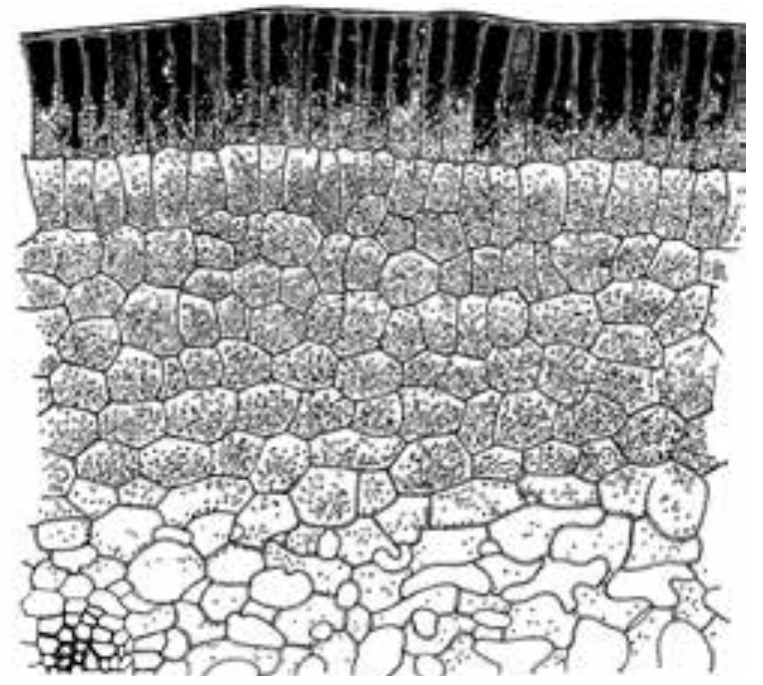

Abb. 10. Gloxinia perennis. Schnitt durch den Osmophor, Zellen unterhalb der Epidermis mit dicht gepackten Reservestoffen (aus Vogel 1966, Abb. 13). 


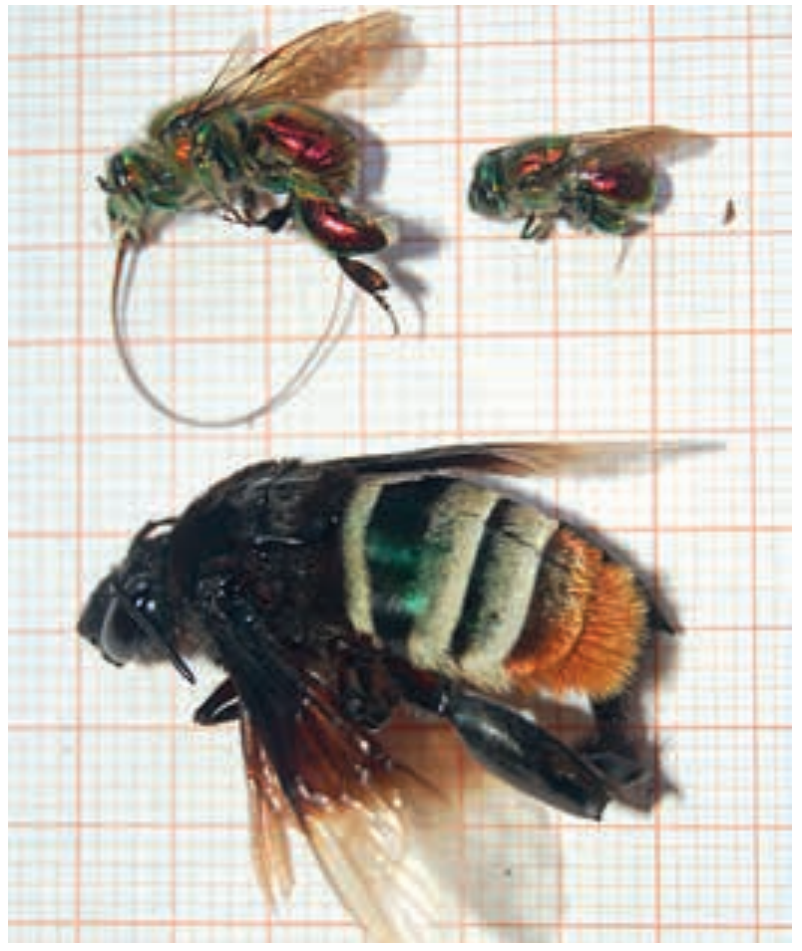

Abb. 11. Zwei Arten von Euglossa (E. cf. flammea, E. alleni) und (unten) die wesentlich größere Eulaema meriana. Jeweils männliche Exemplare (Costa Rica).

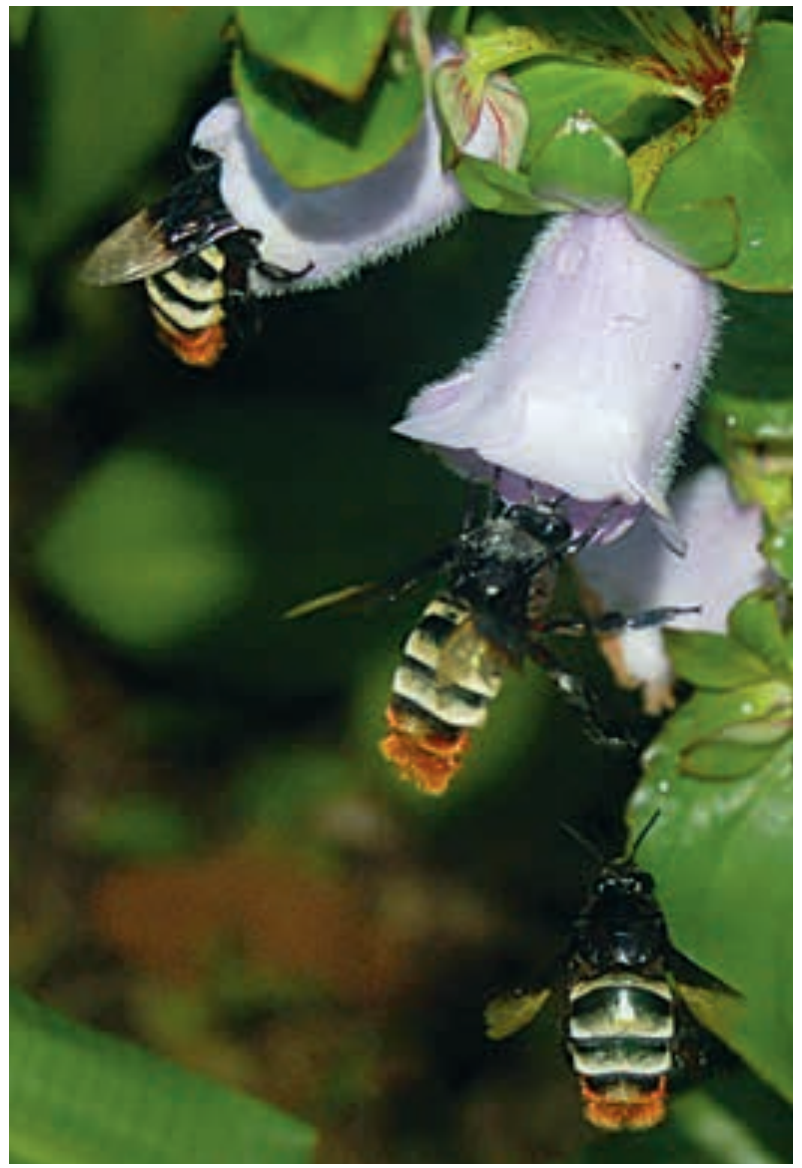

Abb. 12. Gloxinia perennis. Blütenbesuch durch Männchen der Prachtbiene Eulaema meriana in Costa Rica. fen sind. Ihr Blütenbau ist einfach und instruktiv, und eignet sich daher in besonderem Maße, die gegenseitige Anpassung von Parfümblumen an ihre Bestäuber, die männlichen Prachtbienen, zu demonstrieren. Die Blüten sind relativ groß, glockenförmig, schwach dorsiventral und nehmen eine leicht nickende Position ein. Die Farbe der Krone ist bläulich, nur am Blütengrund, der leicht ausgesackt ist, befindet sich ein dunkelvioletter Fleck. Dies ist der Osmophor. Ein Nektarium, wie es in den benachbarten Gattungen vorhanden ist, fehlt. Bei starker Vergrößerung ist zu erkennen, dass die Oberfläche des Osmophors mit feinen Tröpfchen belegt ist, die abgesonderten Duftstoffe. Schnitte durch den Osmophor zeigen, dass das Gewebe zunächst mit Reservestoffen (Stärke) vollgestopft ist. Im Zuge der Sekretionstätigkeit werden diese kontinuierlich abgebaut.

Eine neuere Studie (Witschnig et al. 2008) konnte den von Vogel (1966) beschriebenen Fakten einige weitere Aspekte hinzufügen. Der

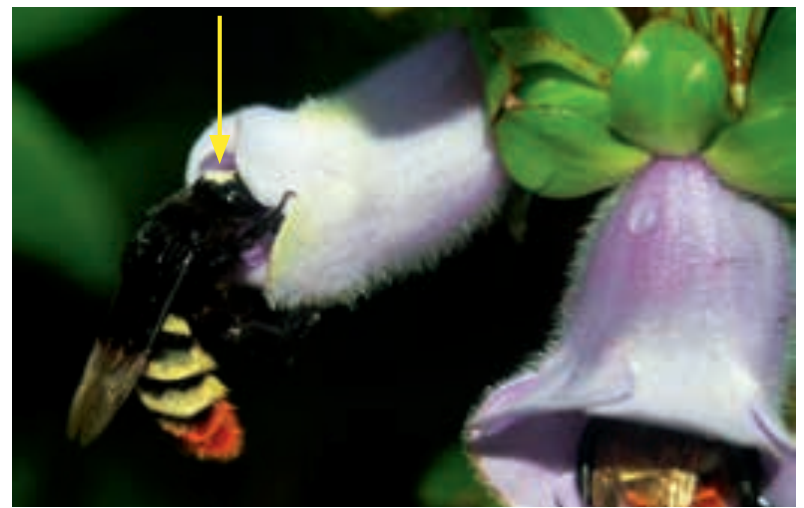

Abb. 13. Landung (links) und Eindringen (rechts) eines Männchens von Eulaema meriana in die Blüte von Gloxinia perennis. Der gelbe Pfeil weist (wie auch in Abb. 14) auf den weißen Gloxinia-Pollen auf der Biene hin.

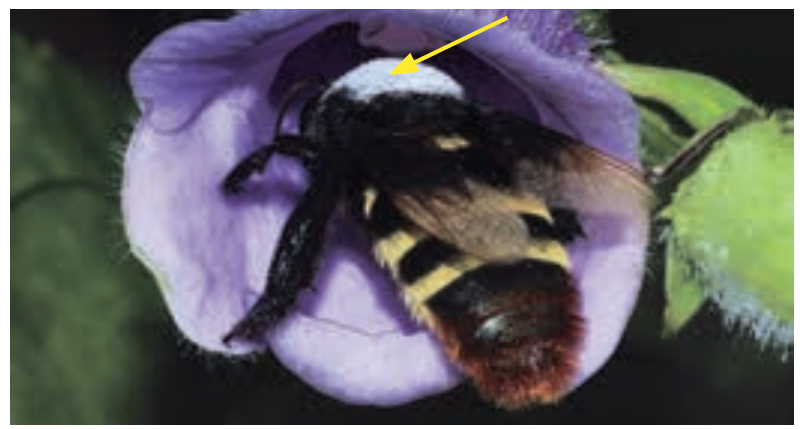

Abb. 14. Blütenbesuch von Gloxinia perennis durch Eulaema meriana im Amazonasgebiet. 
wichtigste ist, dass die Blüten nicht generell an Prachtbienen-Männchen angepasst sind, sondern auf die Arten der Gattung Eulaema. Nur diese Bienen sind groß genug, um beim Sammeln der Duftstoffe die Antheren bzw. Narbe zu berühren. Am Beobachtungsort in Costa Rica erfolgte der Blütenbesuch durch Eulaema meriana knapp vor Tagesanbruch (ca. 5:45 Uhr). Die Männchen von anderen Prachtbienen-Arten trafen gestaffelt zu späteren Stunden am Vormittag ein. Wie die Beobachtungen und Analysen von Video-Filmen zeigte, sind sie wegen ihrer geringen Körpergröße für die Bestäubung irrelevant. Die Studie konnte somit den Nachweis erbringen, dass die Duftstoffe von allen lokalen Prachtbienen wahrgenommen und als attraktiv empfunden wurden, dass aber eine Spezialisierung und Selektierung der Bienen durch die Blütengröße erfolgt. In Peru, in der amazonischen Forschungsstation Panguana, wurde neben Eulaema meriana noch E. cingulata als Bestäuber beobachtet. Diese Prachtbiene ist etwas kleiner als die vorige, berührt aber Staubblätter und Narbe. Vogel selbst beobachtete eine dritte Eulaema-Art in Itatiaia in SO-Brasilien, deutlich ist auf seinen SW-Fotos der Pollenfleck auf dem Rücken der schwarzen $E$. nigrita zu erkennen.

\section{Orchideen}

Die Bestäubungsvorgänge bei den Orchideengattungen Coryanthes, Gongora, Stanhopea und Catasetum gehören zu den spektakulärsten innerhalb der Blütenpflanzen (vgl. Gerlach 1999, 2015, Singer \& Gerlach 2002). Stefan Vogel beobachtete sie bei Catasetum und Gongora in Brasilien. Die ersten Beobachtungen zu Coryanthes finden sich bereits bei CRÜGER (1865), der Direktor am Botanischen Garten in Trinidad war und diese Orchidee vor Ort studieren konnte. CRÜGERs Schlussfolgerungen waren, dass die Prachtbienen an den Blüten „herumknabbern“ würden. Erst 100 Jahre später klärte VoGEL (1963b) die „wahre Funktion des sogenannten Futtergewebes" auf: es handelt sich um Osmophoren.

Die Bestäubungsbiologie der Gattung Coryanthes gehört in der Tat zu den faszinierendsten Tier-Pflanzen-Interaktionen im Pflanzenreich. Die Blüten sind oft faustgroß und besitzen eine Lippe (Labellum) von fleischiger Konsis-

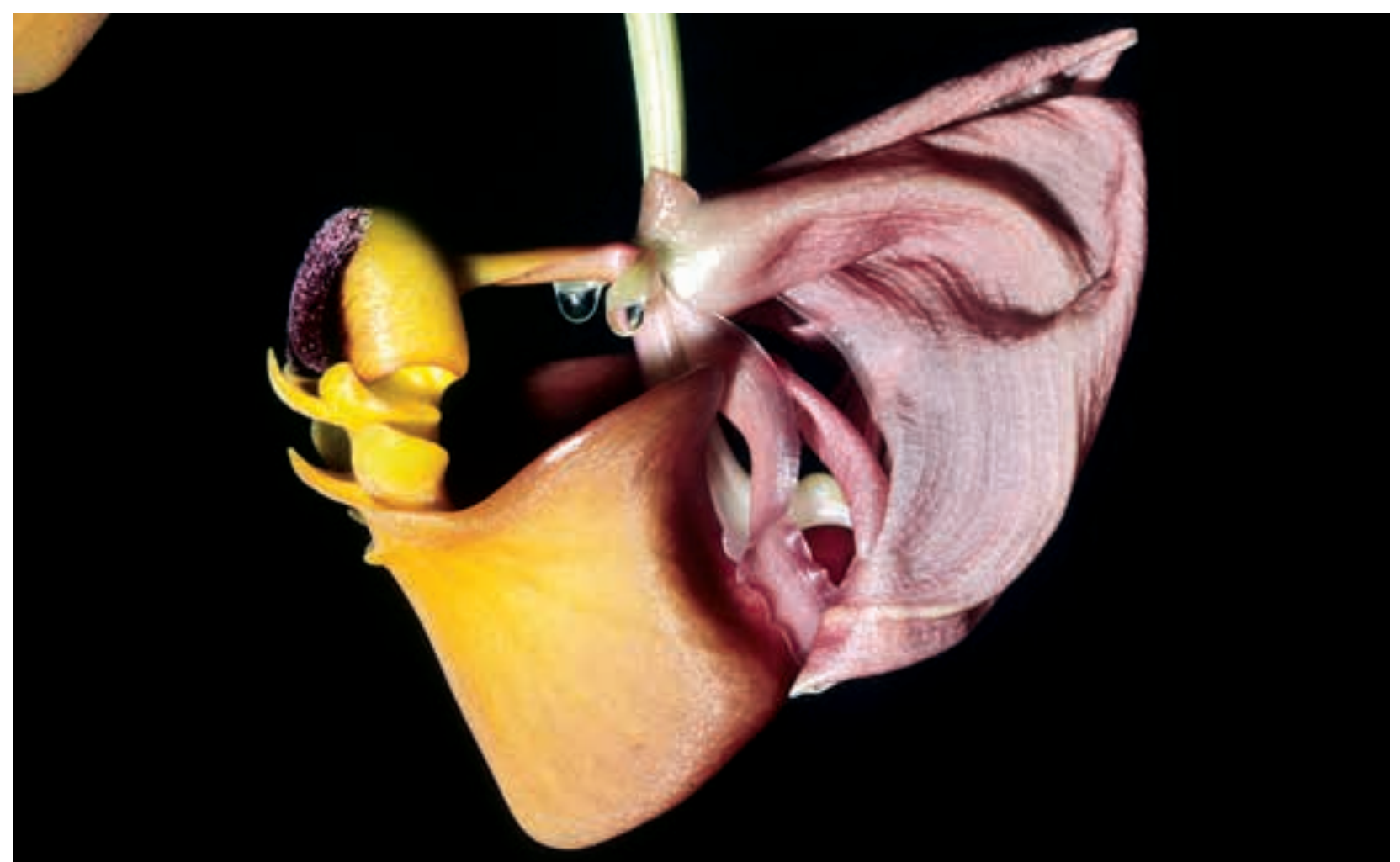

Abb. 15. Blüte von Coryanthes trifoliata. Die Drüsen mit ihren Flüssigkeitstropfen sind gut zu erkennen; unter dem Helm versteckt befinden sich die Osmophoren. 
tenz. Die Lippe ist sehr kompliziert gebaut und feinst abgestimmt auf den passenden Bestäuber. Sie bildet zusammen mit der Säule eine Kesselfalle, die von zwei basalen Drüsen mit einer wässrigen Flüssigkeit gefüllt wird. Das Prachtbienenmännchen landet auf der helmförmigen Basis der Lippe und versucht an die Osmophoren zu gelangen, die sich verdeckt unter dem Helm befinden. Sind die Mops der Vorderbeine gesättigt, muss die Biene, wie bereits geschildert, die Blüte verlassen. Beim Umfliegen der Blüte kommt sie eventuell mit einem der zwei Tropfen der basalen Drüsen in Berührung und fällt wie ein Stein in die Kesselfalle. Da sie wegen der benetzten Flügel nicht mehr flugfähig ist, kann das Tier nun nicht mehr fliegend entkommen. Auch krabbelnd besteht keine Chance, das Bad zu verlassen, da die Wände äußerst glatt und von ähnlicher Struktur wie bei den fleischfressenden Kannenpflanzen sind. Nach einiger Zeit erkennt das Tier eine sich über den Flüssigkeitsspiegel erhebende Rettungsinsel. Es schiebt sich mühsam darauf und gelangt in einen Tunnel, gebildet von der Lippe und der Säule. Dieser Ausgang ist etwas niedriger als das Insekt, es muss sich

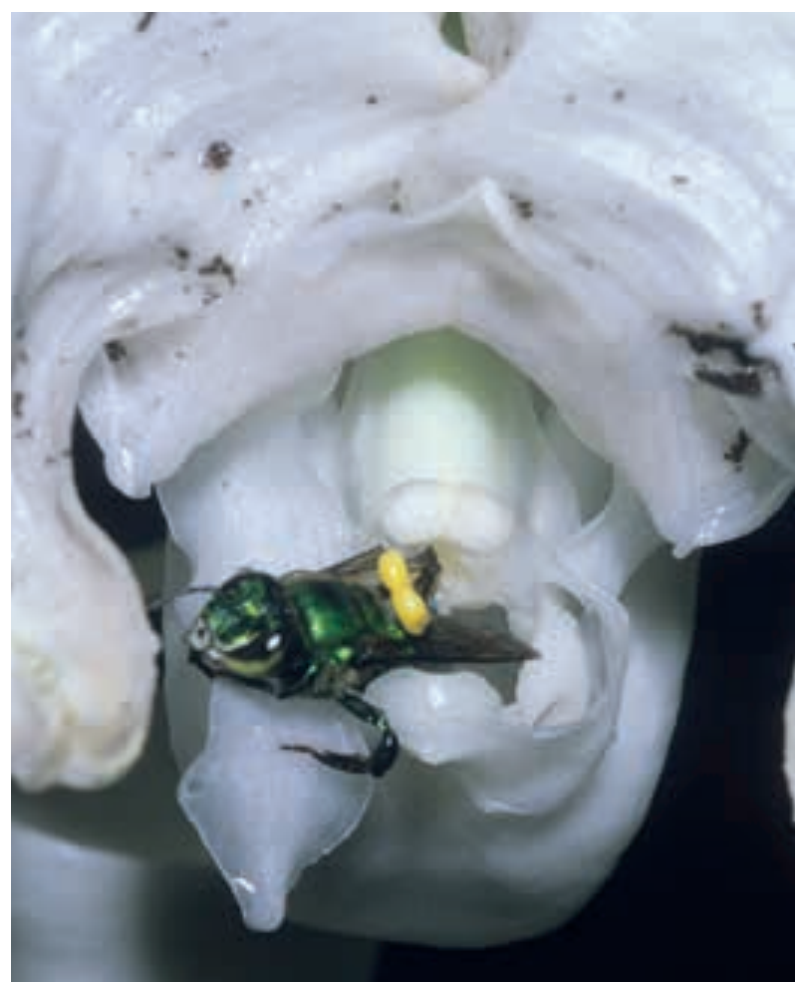

Abb. 17. Euglossa orellana beim Verlassen der Blüte von Coryanthes vasquezii; deutlich sind die gelben Pollinien auf der Biene zu erkennen (Bolivien).

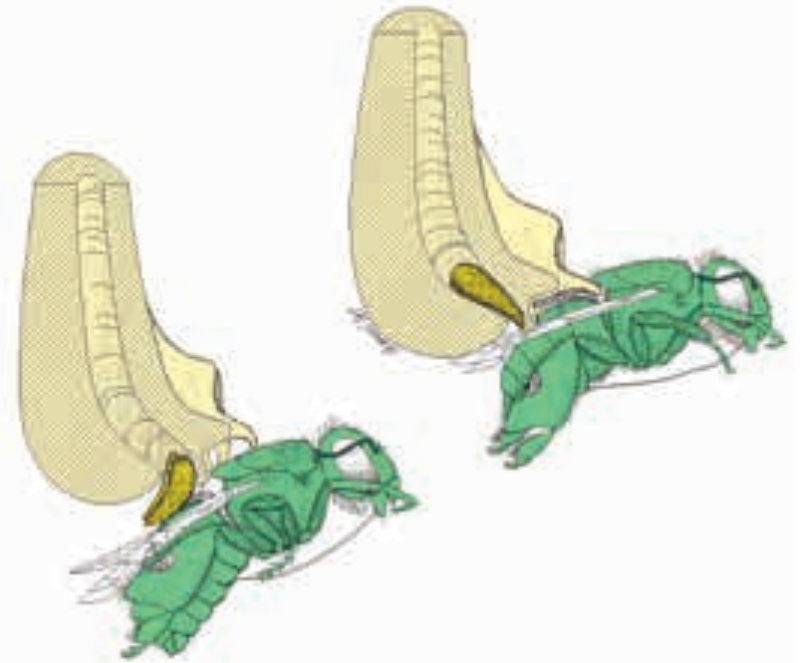

Abb. 16. Beim Verlassen einer Coryanthes-Blüte muss das Prachtbienenmännchen einen engen Kanal passieren, hier schiebt es die Pollinien in den Narbenkanal die dann durch die Vorwärtsbewegung des Tiers vom restlichen Pollinarium abgerissen werden (aus Nazarov \& Gerlach 1997, Fig. 5).

mit Kraft durch den Engpass drücken, was durch eine elastische Aufhängung der Lippe ermöglicht wird. Bei der weiteren Vorwärtsbewegung passiert das Prachtbienenmännchen zunächst die Narbe, und kurz vor dem Verlassen der Blüte wird ihm schließlich das Pollinarium (bei den Orchideen

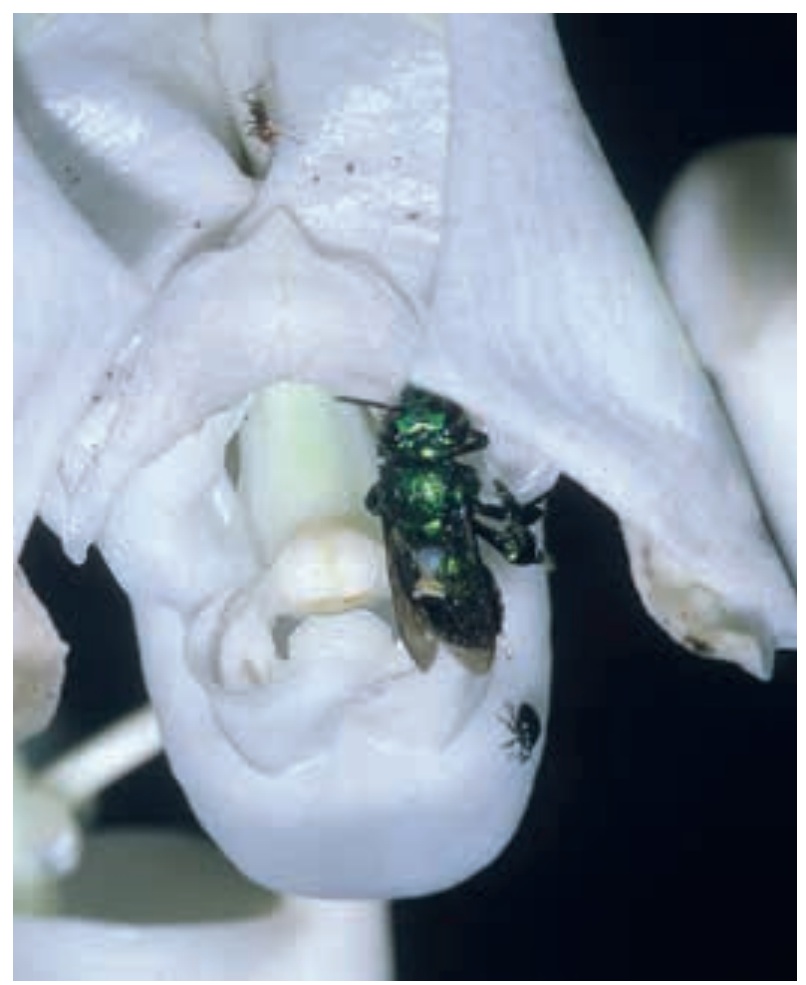

Abb. 18. Dieses Exemplar von Euglossa orellana hat die Blüte von Coryanthes vasquezii bestäubt; auf dem Bienenkörper haften noch die Reste des Pollinariums. 
ist der Pollen des einzigen Staubblattes zu den sogenannten Pollinien verbacken und wird mitsamt dem Stielchen und einem Klebeapparat als Pollinarium dem Bestäuber angeheftet) in die Taille geklebt. Dieser Vorgang dauert zuweilen bis zu 10 Minuten, sodass das Tier von der Kraftanstrengung völlig erschöpft ist. Es ruht zunächst etwas und trocknet sich, bevor es weiterfliegen kann. Das Bad war so unangenehm, dass die Prachtbiene nicht sofort weitere Blüten anfliegt. Durch die Trocknung schrumpft das Pollinarium etwas und passt erst dann in den schmalen Narbenspalt (Verhindern von Selbstbestäubung). Sind nach einiger Zeit die unangenehmen Erfahrungen vergessen und der Duftsammeltrieb unbezähmbar geworden, besucht das Bienenmännchen eine weitere Blüte. Dort widerfährt ihr das gleiche Schicksal. Sie muss wieder ein unfreiwilliges Bad nehmen und sich durch den engen Tunnel zwängen. Bei dieser erneuten Passage drückt es die Pollinarien in den Narbenspalt. Dabei schiebt sich eine gabelförmige Struktur an der Säulenspitze zwischen das Stielchen und die

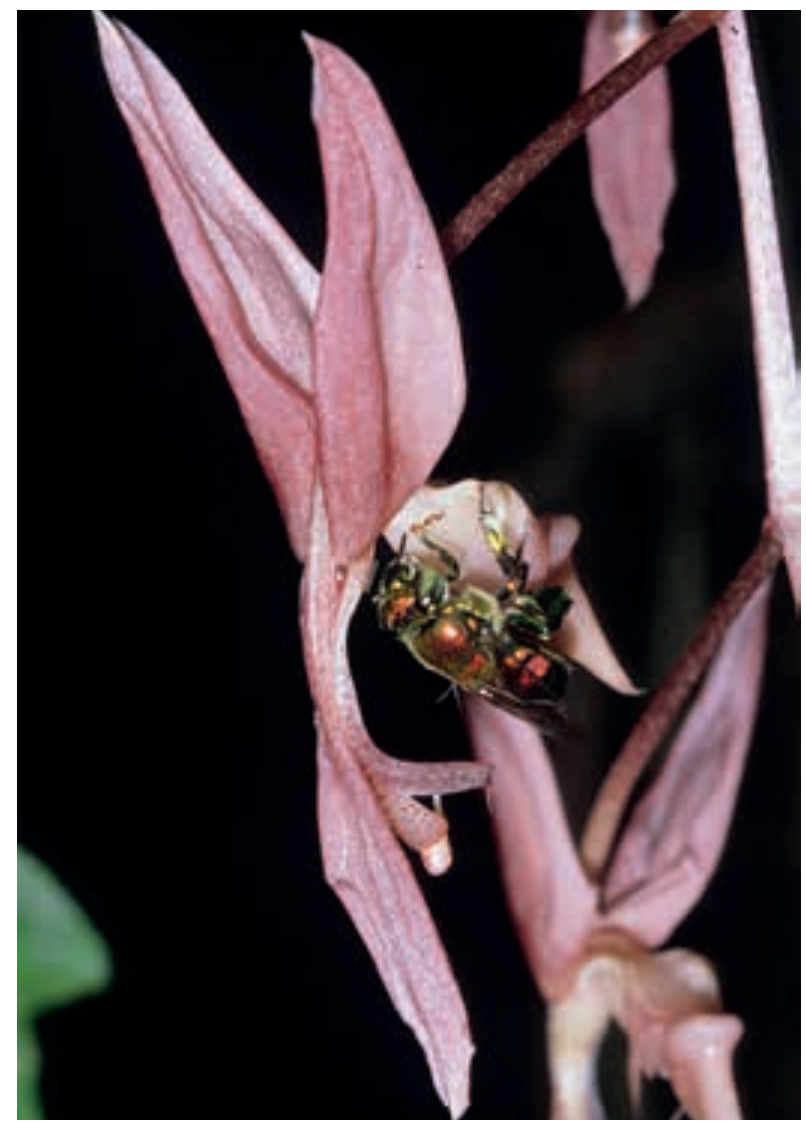

Abb. 19. Gongora unicolor mit duftsammelndem Männchen von Euglossa alleni (Costa Rica).
Pollinien des Pollinariums. Durch die Vorwärtsbewegung des Tiers werden dabei die Pollinien vom restlichen Pollinarium abgerissen.

Die Coryanthes-Arten besitzen die schwersten Blüten der Orchideen, sie sind aber mit zwei bis vier Tagen Lebensdauer nur sehr kurzlebig. Das Zusammenspiel zwischen der Blüte und dem bestäubenden Prachtbienenmännchen ist sehr effektiv, deshalb reichen die wenigen Tage aus, um für genügend Samenproduktion zu sorgen. Von den Blüten wird meist nur eine einzige Prachtbienen-Art angelockt. Die Größe der Biene und der Blütenteile sind exakt aufeinander abgestimmt. Der Tunnelquerschnitt ist wie bereits gesagt etwas kleiner als der des Bestäubers, der sich nur mit Mühe durch den engen Ausgang zwängen kann. Würde eine zu große Bienen-Art in die Kesselfalle geraten, so könnte sie nicht aus der Blüte gelangen, würde ertrinken und den Ausgang blockieren, tragisch für Tier und Blüte. Eine zu kleine Prachtbienen-Art könnte passieren, ohne dass ihr das Pollinarium aufgeklebt würden, sie wäre ebenfalls nicht der Bestäubung dienlich.

Blüten der Gattung Gongora werden von den drei nicht parasitischen Gattungen Euglossa, Eufriesea (bei Vogel noch Euplusia genannt) und Eulaema bestäubt. Die Lippe der verschiedenen Gongora-Arten ist sehr komplex gebaut, sie steht in natürlicher Position über der Säule. Die Prachtbienenmännchen landen auf ihr und hängen kopfüber an ihr, um an die Duftstoffquelle an ihrer Basis zu gelangen. Das Auffliegen, um die gesammelten Duftstoffe in die Hinterbeine zu transferieren, gestaltet sich für die Tiere so schwieriger. Sie lassen sich fallen, rutschen mit dem Rücken entlang der Säule, werden von den seitlich mit der Säule verwachsenen Petalen zentriert und passieren so zunächst die Narbe und dann die Anthere. So wird das Pollinarium der Biene zwischen Thorax und Abdomen geklebt. Die Petalen haben dabei die Funktion eines Geländers, welches ein seitliches Abgleiten von der Rutschbahn verhindert. Zunächst ist die schlitzförmige Narbe weitgehend geschlossen, sie öffnet sich erst, wenn das Pollinarium entfernt wurde. So wird effektiv eine Selbstbestäubung vermieden. 
Gelangt eine mit einem Gongora-Pollinarium belegte Prachtbiene auf die Rutschbahn, so nimmt die Narbe die Pollinien auf und reißt sie durch die Bewegung der Biene vom restlichen Pollinarium (Klebescheibe plus Stielchen) ab. Somit ist die Bestäubung vollzogen.

Die Blüten der Stanhopea-Arten sind von fleischiger Konsistenz, die Blütenstände sind wie bei den Gongora-Arten hängend. Die Lippe ist vertikal orientiert. Sie besitzt an der Basis eine Höhlung, welche sackartig vertieft ist. Darin befindet sich der Osmophorenbereich. Das am Blütenduft interessierte Prachtbienenmännchen fliegt die Blüte seitwärts an und gelangt in die Nähe der Höhlung. Es hat dort ein Problem mit dem Festhalten, denn die ganze Lippenoberfläche ist sehr glatt. Mühsam dringt es in die Höhlung ein und wischt mit den ausgestreckten Vorderbeinen die Oberfläche der Osmophoren ab. Zum Abheben und Rückladen verlässt es die Höhlung, stößt gegen die Säule, rutscht an dieser entlang und wird ebenfalls von einem „Geländer“, gebildet von Lippenanhängen,

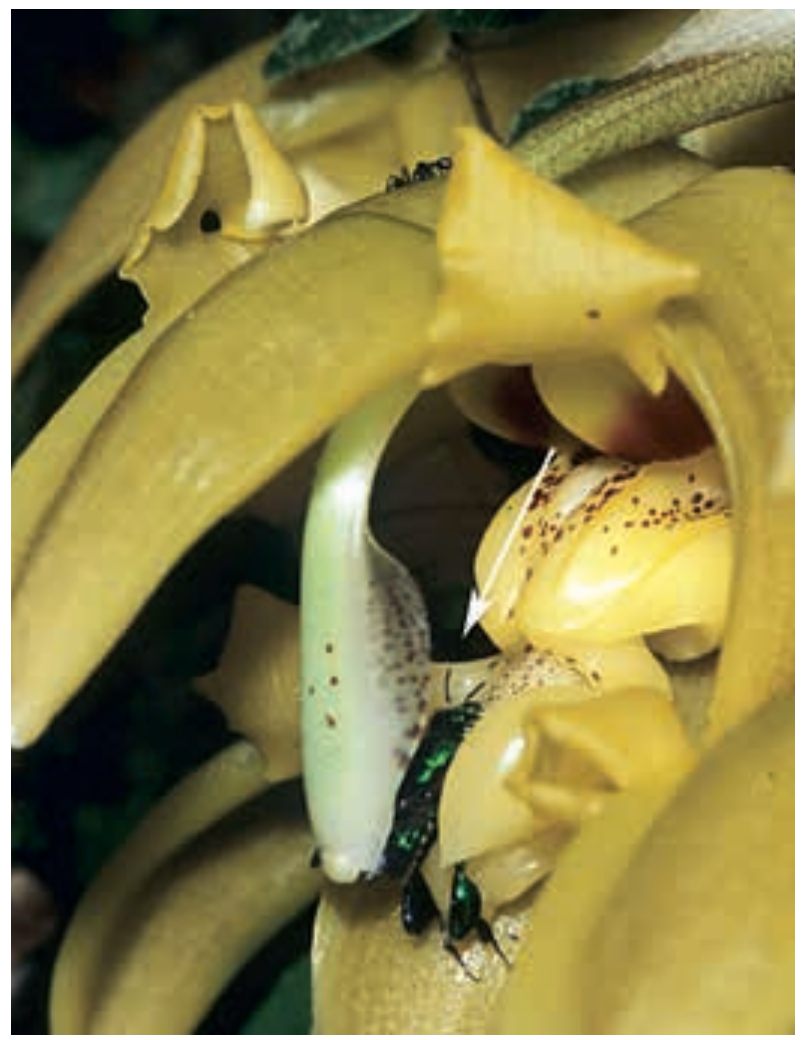

Abb. 20. Beim Besuch von Stanhopea-Blüten (hier S. anfracta) rutscht das Euglossa-Männchen in Pfeilrichtung durch die Engstelle von Labellum und Säule und wird dort mit dem Pollinarium beladen (Ecuador). zentriert. Beim weiteren Fall bleibt es am Rostellum (einem Teil der Säule) hängen, welches sich zwischen Thorax und Abdomen schiebt und bekommt dort das Pollinarium aufklebt. Nun rutscht es weiter an der Narbe vorbei oder entkommt nach oben. Wurde das Pollinarium bereits von einer anderen Biene entfernt, rutscht die Biene nach unten an der Narbe vorbei und ein eventuell mitgebrachtes Pollinarium wird so in den Narbenspalt geschoben, wodurch es zur Bestäubung kommt.

Die mehr als 170 Catasetum-Arten weisen eingeschlechtliche Blüten an ebensolchen Blütenständen auf, eine Rarität bei den Orchideen. Die männlichen Blüten sind äußerst vielgestaltig. Hingegen sind die weiblichen Blüten einförmig, sodass sich an ihnen die Arten nicht unterscheiden lassen. Sie besitzen eine haubenförmige Lippe, welche über der Säule steht. Die Lippen der männlichen Blüten stehen meist unter der Säule, sie können u. a. flach, haubenförmig, gefranst oder gezähnt sein, sie sind oft komplex aufgebaut. Den männlichen Blüten gemein sind zwei berührungsempfindliche Antennen,

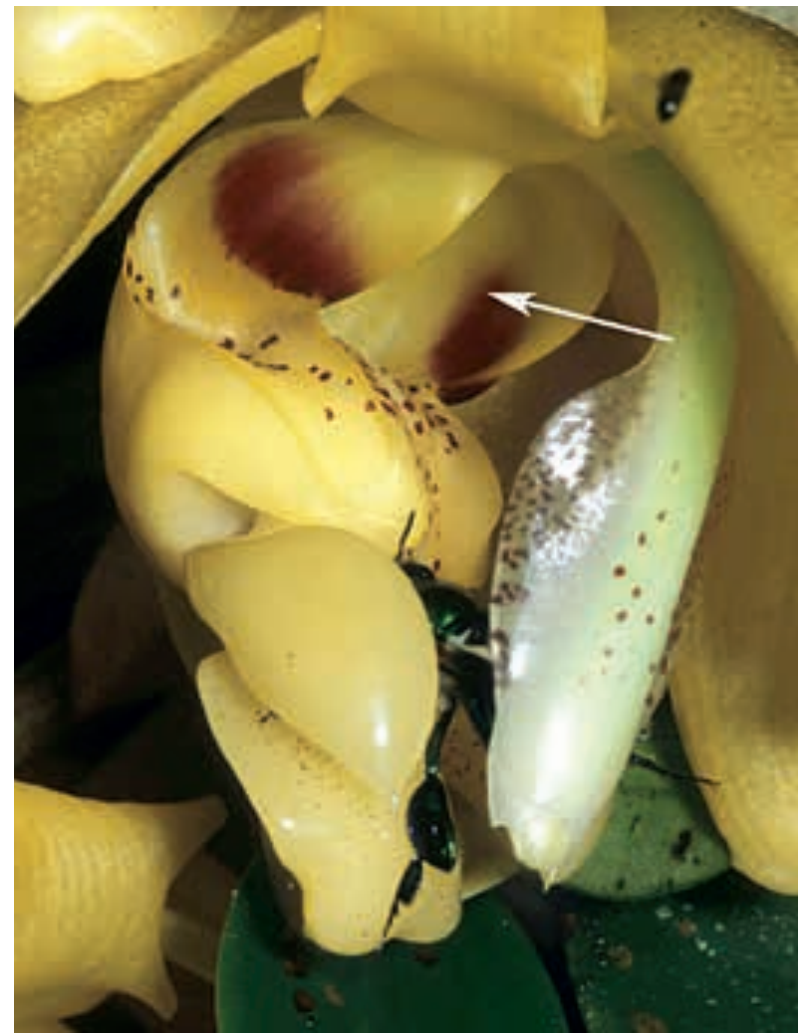

Abb. 21. Das Tier wurde gerade mit einem Pollinarium belegt; gut ist das lange, weiße Stielchen zu erkennen. Der Pfeil weist in die Lippenhöhlung, in der sich der Osmophorenbereich befindet. 


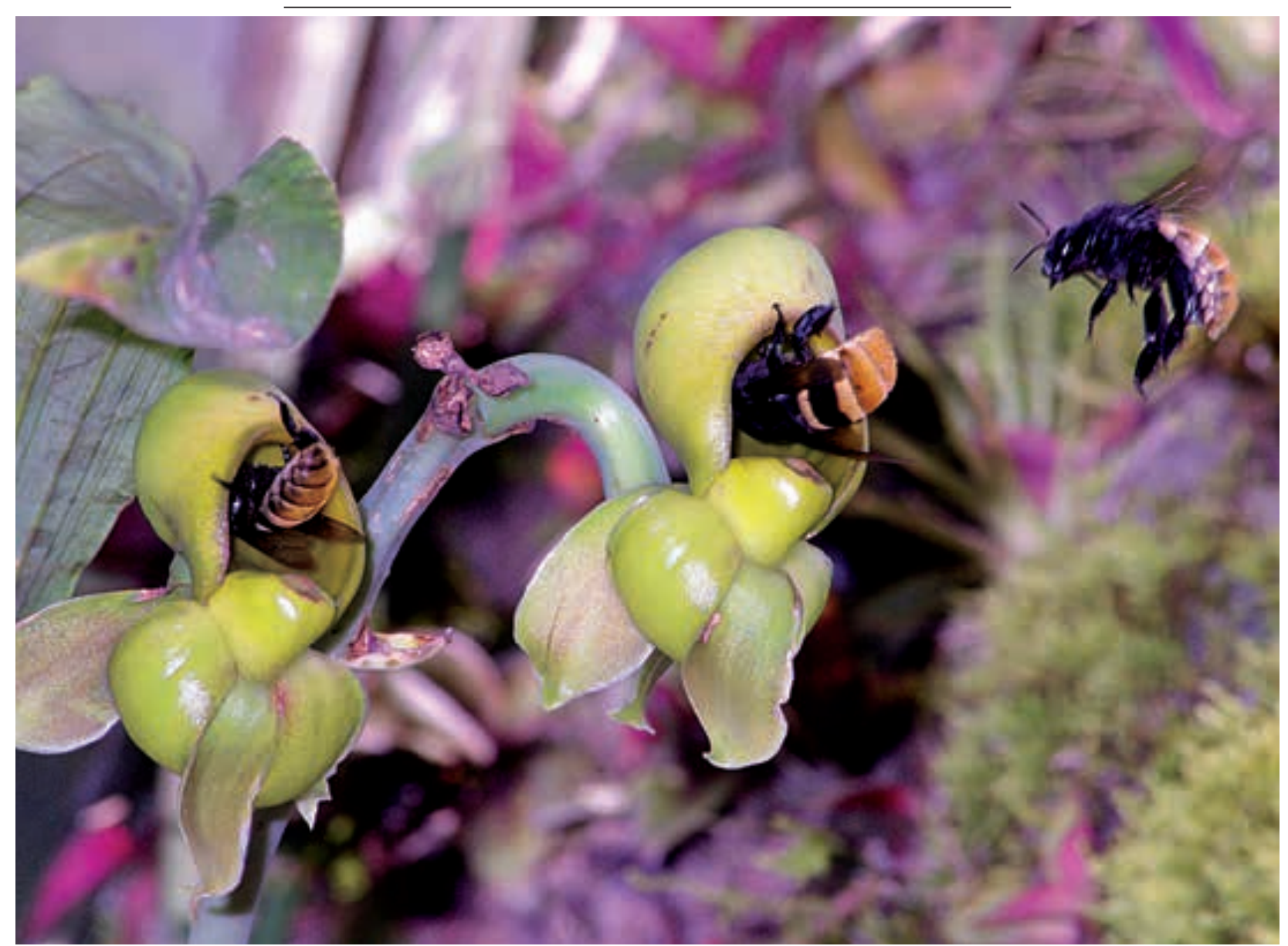

Abb. 22. Männchen der Prachtbiene Eulaema cingulata beim Besuch der weiblichen Blüten von Catasetum maculatum (Orchidaceae) (Costa Rica).

die entweder gleichförmig nebeneinander liegen oder deren Eine bogenförmig unter die Andere gekrümmt ist. Diese Antennen liegen über der Lippe, sie sind Auswüchse der Säule. Wie bei den zuvor ausgeführten Fällen liegt der Osmophorenbereich an der Basis der Lippe. Das Prachtbienenmännchen landet auf dieser und stößt irgendwann beim Duftsammeln an eine der Antennen, blitzartig wird ihm das Pollinarium zielgenau auf den Rücken geschleudert. Vom Aufprall erschreckt verlässt das Tier die Blüte und fliegt mit dem Pollinarium davon. Gelangt eine Pollinarium-tragende Biene an eine weibliche Blüte, so krabbelt sie an den Osmophorenbereich in die Haube der Lippe, das Pollinium baumelt wegen der Schwerkraft über der Narbe. Beim Verlassen der Blüte gelangt es in den Narbenspalt und wird abgestreift. Was ist nun der Vorteil der eingeschlechtlichen Blüten? Es wird auf jeden Fall Fremdbestäubung gewährleistet.

Was ist aber die treibende Kraft, welche die Biene von der männlichen zur weiblichen Blüte bewegt?
Der Schock durch den gewaltigen Aufprall muss wohl sehr tief sitzen, denn sehr selten werden Bienen mit mehr als einem Pollinarium von Catasetum gefunden, während das z.B. bei Gongora-Arten häufig der Fall ist. Vermeidet die Biene nun rein visuell einen weiteren Besuch einer männlichen Blüte? Untersuchungen des Blütenduftes von männlichen und weiblichen Blüten zeigen einen weitgehend gleichartigen Blütenduft, so dass olfaktorische Differenzen wohl nicht die Entscheidungen des Bestäubers beeinflussen dürften. Die Erforschung dieses Sachverhaltes ist noch im vollen Gange und kann nur im natürlichen Habitat aufgeklärt werden. Experimentelle Beweise des Verhaltens der Bestäuber an den Catasetum-Blüten sind schwierig zu führen und sehr zeitaufwendig. STEFan Vogel beobachtete akribisch und mit unendlicher Geduld die Vorgänge an den Blüten und studierte mit einmaliger Genauigkeit die involvierten Organismen in allen Details. Durch seine umfassende Bildung konnte er Zusammenhänge erkennen, die anderen Forschern vor und neben ihm verborgen blieben. 


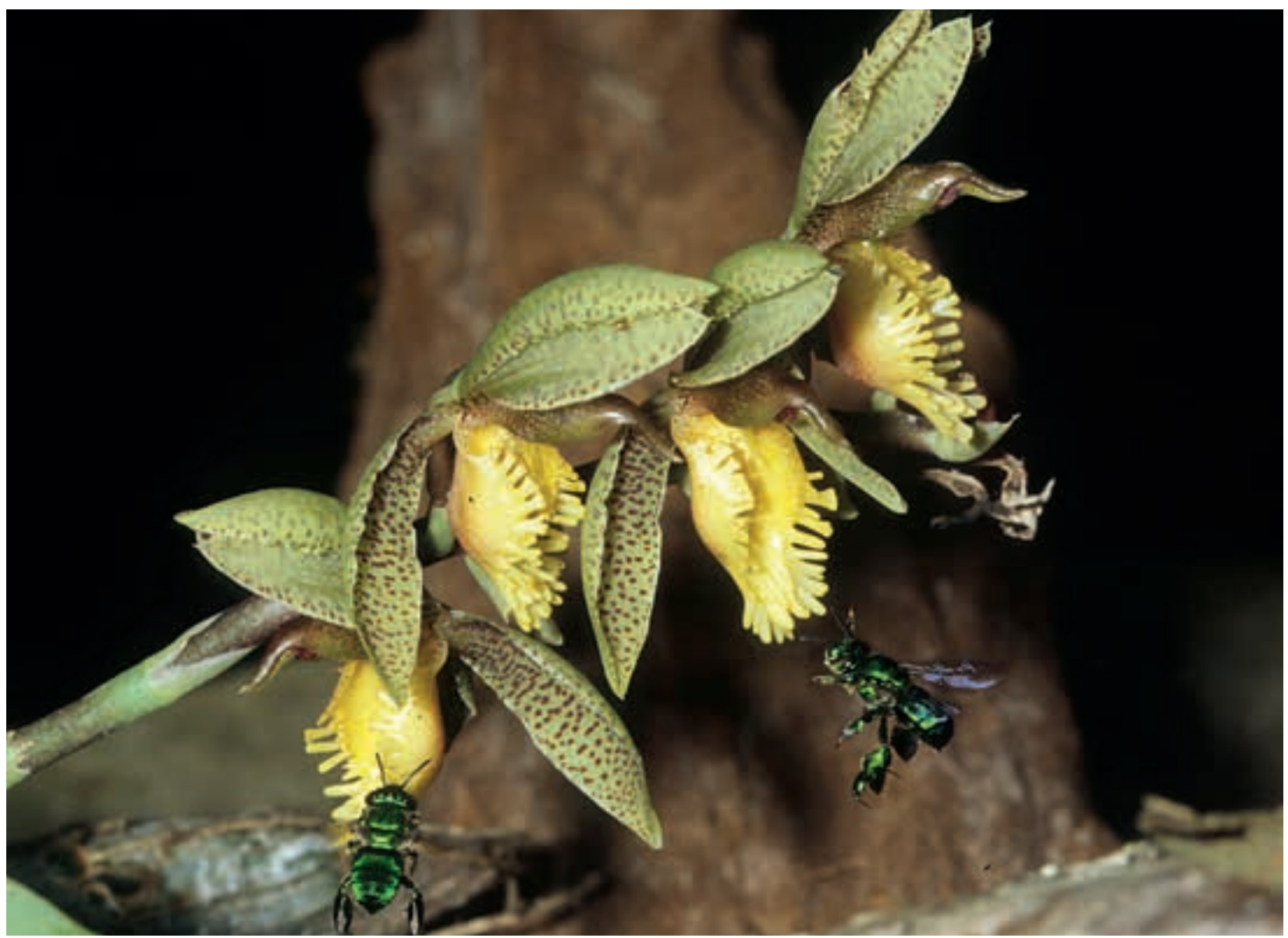

Abb. 23. Männliche Blüten von Catasetum schunkei mit Bestäubern (Euglossa augaspis) (Peru).

\section{Literatur}

Armbruster, W. S. \& Webster, G. L. 1979: Pollination of two species of Dalechampia (Euphorbiaceae) in Mexico by euglossine bees. - Biotropica 11: 278-283.

Boggan, J. K. 2006: What happened to Gloxinia? - Gesneriads 56: 26-32.

CRÜGER, H. 1865: A few notes on the fecundation of orchids and their morphology. - Bot. J. Linn. Soc. 8: 127-135.

Dodson, C. H. \& Frymire, G. P. 1961: Natural pollination of orchids. - Missouri Bot. Gard. Bull. 49: 133-152.

Eltz, T. 2003: Duftakkumulation bei Prachtbienen (Euglossini): Mechanismen, Muster, offene Fragen. - Verhandlungen Westdeutscher Entomologentag 15: 11-30.

Eltz, T. 2010: Sag's durch die Blume: Evolution des Parfümsammelns bei Prachtbienen (Euglossini). - Entomologie heute 22: 3-16.

Gerlach G. 1999: Mit allen Tricks - über Strategien von Orchideen. - In: Zizka, G., Schneckenburger, S. (Hrsg.): Blütenökologie - faszinierendes Miteinander von Pflanze und Tieren. - Kleine Senckenberg-Reihe 33 - Palmengarten Sonderheft Nr. 31: 135-140.
Gerlach, G. 2015: Prachtbienen und Orchideen - eine ökologische Besonderheit der Neotropis. - In: Feest, C., Kron, C. (Hrsg.): Regenwald. Begleitbuch zur Sonderausstellung Ausstellungszentrum Lokschuppen, Rosenheim, 44-49.

Hentrich, H., Kaiser, R., Gottsberger, G. 2010: Floral biology and reproductive isolation by floral scent in three sympatric aroid species in French Guiana. - Plant Biol. 12: 587-596.

Nazarov, V. V., Gerlach, G. 1997: The potential seed productivity of orchid flowers and peculiarities of their pollination systems. - Lindleyana 12: 188-204.

Roalson, E. H., Boggan, J. K., Skog, L. E., Zimmer, E. A. 2005a: Untangling the Gloxinieae (Gesneriaceae). I. Phylogenetic patterns and generic boundaries inferred from nuclear, chloroplast, and morphological cladistic data sets. Taxon 54: 389-410.

Roalson, E. H., Boggan, J. K., Skog, L. E. 2005b: Reorganization of tribal and generic boundaries in the Gloxinieae (Gesneriaceae: Gesnerioideae) and the description of a new tribe in the Gesnerioideae, Sphaerorrhizeae. - Selbyana 25: 225-238.

Sazima, M., Vogel, S., Cocucci, A., Hausner, G. 1993: The perfume flowers of Cyphomandra (Solanaceae): Pollination by euglossine bees, bellows mechanism, osmophores, and volatiles. - Plant Syst. Evol. 187: 51-88. 
Schwerdtfeger, M., Gerlach, G., Kaiser, R. 2002: Anthecology in the Neotropical genus Anthurium (Araceae) - a preliminary report. - Selbyana 23: 258-267.

Singer, R., Gerlach, G. 2002: Prachtbienen und Orchideen - Neue Erkenntnisse und Anmerkungen zu Südost-Brasilien. Journal für den Orchideenfreund 9: 139-149.

Teichert, H., Dötterl, S., Zimma, B., Ayasse, M., Gottsberger, G. 2009: Perfume-collecting male euglossine bees as pollinators of a basal angiosperm: the case of Unonopsis stipitata (Annonaceae). - Plant Biol. 11: 29-37.

Troll, W. 1961: Kommission für biologische Forschung. Bericht. - Jahrb. Akad. Wiss. Lit. Mainz.

Vogel, S. 1963a: Duftdrüsen im Dienste der Bestäubung. Über Bau und Funktion der Osmophoren. - Abhandl. Akad. Wiss. Lit. Mainz, Math.-Naturwiss. Kl. 10: 600-763.

Vogel, S. 1963b: Das sexuelle Anlockungsprinzip der Catasetineen- und Stanhopeen-Blüten und die wahre Funktion ihres sogenannten Futtergewebes. - Österr. Bot. Z. 110: 308-337.

Vogel, S. 1966: Parfümsammelnde Bienen als Bestäuber von Orchideen und Gloxinia. - Österr. Bot. Z. 113: 302-361.

Vogel, S. 1967: „Parfümblumen“ und parfümsammelnde Bienen. - Umschau Wiss. Techn. 67: 327.
Vogel, S. 1999: Von Ölblumen und Parfümblumen. - In: Zizka, G., Schneckenburger, S. (Hrsg.): Blütenökologie faszinierendes Miteinander von Pflanzen und Tieren. - Kleine Senckenberg-Reihe Nr. 33. Palmengarten Sonderheft Nr. 31: 74-87.

Vogel, S., Weber, A. Huber, W. 2005: Perfume flowers in Gesneriaceae and their pollination by euglossine bees. Gloxinian 55: 46-53.

Weber, A. \& Steinecke, H. 2017: Die großen wissenschaftlichen Leistungen von Stefan Vogel (1925-2015). Teil 3. Blütendüfte und ihre Bildung in den Osmophoren. - Palmengarten 81: 69-77.

Webster, G. L., Armbruster, W. S. 1991: A synopsis of the neotropical species of Dalechampia (Euphorbiaceae). - Bot. J. Linn. Soc. 105: 137-177

Witschnig, G., Hickl, C., Weber, A. 2008: Notes on the pollination of the perfume flowers of Gloxinia perennis (Gesneriaceae) by euglossine bees. - In: Weissenhofer, A., Huber, W., Mayer, V., Pamperl, S., Weber, A., Aubrecht, G. (eds.): Natural and cultural history of the Golfo Dulce region, Costa Rica. Historia natural y cultural de la region del Golfo Dulce, Costa Rica. - Linz: O.Ö. Landesmuseum, Stapfia 88: 573-578.
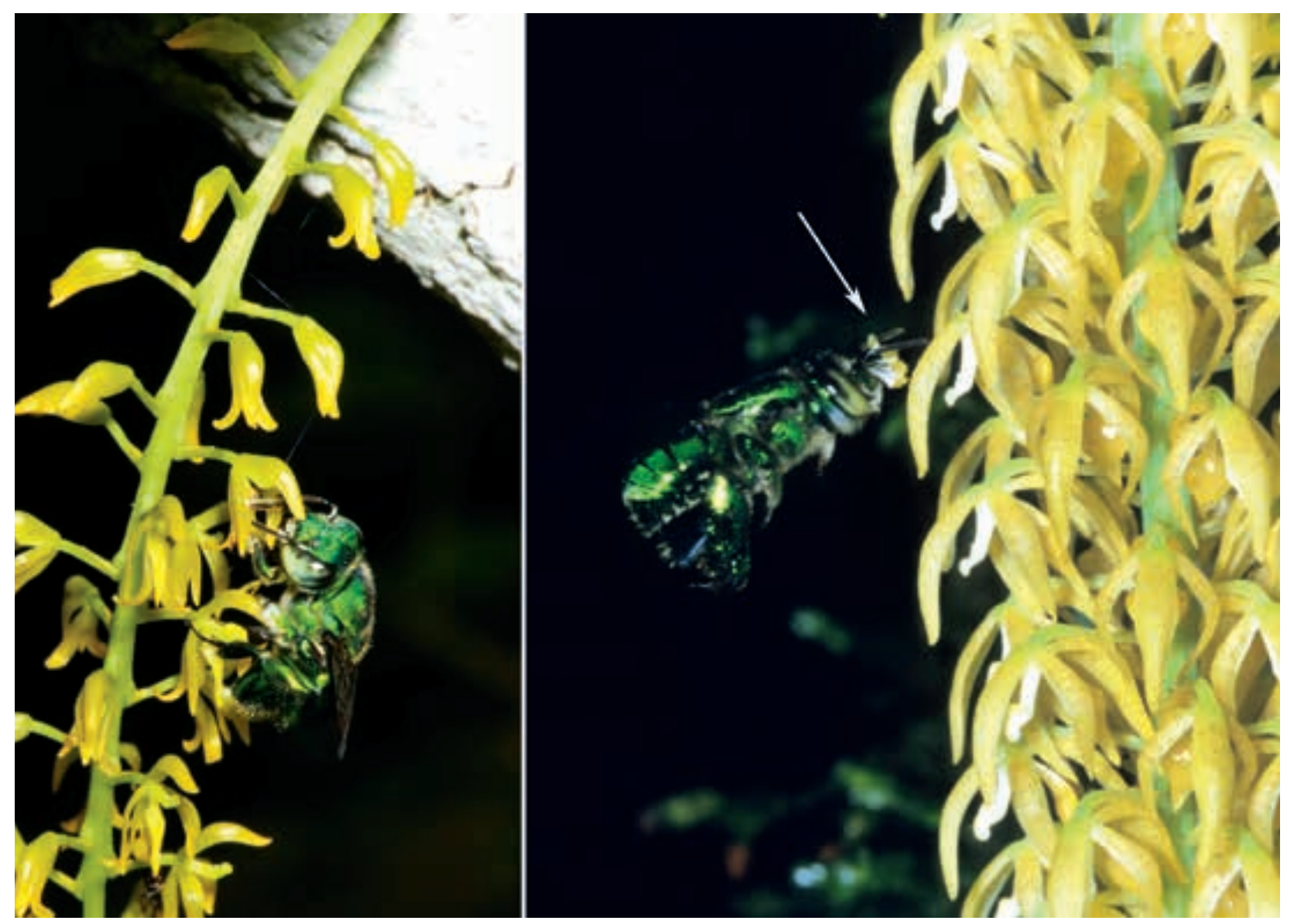

Abb. 24. Bei Notylia-Arten (Orchidaceae) ist der Bestäuber deutlich größer als die Blüte. Trotzdem werden ihm die Pollinarien exakt ins Gesicht geklebt. Links: Notylia sp. mit Euglossa sp. (Brasilien). Rechts: Notylia buchtienii mit Euglossa augaspis (Bolivien). Der Pfeil zeigt auf das Büschel von Pollinarien im Gesicht der Biene. 